\title{
THE NUMERICAL COMPUTATION OF HETEROCLINIC CONNECTIONS IN SYSTEMS OF GRADIENT PARTIAL DIFFERENTIAL EQUATIONS*
}

\author{
FENGSHAN BAI ${ }^{\dagger \ddagger}$, ALASTAIR SPENCE ${ }^{\dagger}$, AND ANDREW M. STUART ${ }^{\dagger \S}$
}

\begin{abstract}
The numerical computation of heteroclinic connections in partial differential equations (PDEs) with a gradient structure, such as those arising in the modeling of phase transitions, is considered. Initially, a scalar reaction diffusion equation is studied; structural assumptions are made on the problem to ensure the existence of absorbing sets and, consequently, a global attractor. As a result of the gradient structure, it is known that, if all equilibria are hyperbolic, the global attractor comprises the set of equilibria and heteroclinic orbits connecting equilibria to one another. Thus it is natural to consider direct approximation of the set of equilibria and the connecting orbits.

Results are proved about the Fourier spanning basis for branches of equilibria and also for certain heteroclinic connections; these results exploit the oddness of the nonlinearity. The reaction-diffusion equation is then approximated by a Galerkin spectral discretization to produce a system of ordinary differential equations (ODEs). Analogous results to those holding for the PDE are proved for the ODEs -in particular, the existence and structure of the global attractor and appropriate spanning bases for the equilibria and certain heteroclinic connections, are studied. Heteroclinic connections in the system of ODEs are then computed using a generalization of known methods to cope with the gradient structure. Suitable parameterizations of the attractor are introduced and numerical continuation used to find families of connections on the attractor. Special connections, which are stable in certain Fourier spanning bases, are used as starting points for the computations.

The methods used allow the calculation of connecting orbits that are unstable as solutions of the initial value problem, and thus provide a computational tool for understanding the dynamics of dissipative problems in a manner that could not be achieved by use of standard initial value methods. Numerical results are given for the Chafee-Infante problem and for the Cahn-Hilliard equation. A one-parameter family of PDEs connecting these two problems is introduced, and it is demonstrated numerically that the global attractor for the Chafee-Infante problem can be continuously deformed into that for the Cahn-Hilliard equation.
\end{abstract}

Key words. infinite-dimensional dynamical systems, global attractor, heteroclinic connections, gradient systems, reaction-diffusion equations, Galerkin spectral method, invariant subspaces, continuation

AMS(MOS) subject classifications. $65 \mathrm{~N}, 65 \mathrm{~L}, 34 \mathrm{~B}, 35 \mathrm{P}$

1. Introduction. The large time dynamics of certain classes of dissipative, infinite-dimensional, dynamical systems are captured in the global attractor [26], [23], [17], [18]. This is an invariant set that comprises all the complete orbits of the dynamical system, which are defined backward and forward in time-that is, heteroclinic or homoclinic orbits connecting $\alpha$ and $\omega$ limit sets. It is thus natural to consider direct approximation of these connecting orbits as a means to understanding the large time dynamics of a system; in particular, it is of interest to find connections that are unstable as solutions of the initial value problem but that play an important role in determining the long time dynamics. In this paper, we consider the particular case of dissipative partial differential equations (PDEs) that have a gradient structure; it is then known that, provided that the equilibria are hyperbolic, the global attractor

* Received by the editors January 13, 1992; accepted for publication (in revised form) June 5, 1992. This work was supported by the United Kingdom Science Engineering Research Council.

$\dagger$ School of Mathematical Sciences, University of Bath, Bath BA2 7AY, United Kingdom.

$\ddagger$ The research of this author was performed while he was on leave from Applied Mathematics Department, Tsinghua University, Beijing 100084, People's Republic of China.

$\S$ Present address, Division of Applied Mechanics, Program in Scientific Computing and Computational Mathematics, Durand-252, Stanford University, Stanford, California 94305. 
comprises the union of equilibrium points together with heteroclinic orbits connecting them to one another (see [17], [26]).

Gradient systems arise in many areas of application where there is a dynamic energy minimization process together with multiple competing equilibria. One particular example is the field of phase transitions where the Cahn-Hilliard [4] and phase-field [3] models have been very successful and have stimulated a great deal of practical and theoretical interest. In these problems, the nondimensional transient timescales are frequently very long, due to the fact that the timescale for penalization of interfacial energy between two phases is long relative to the chemical relaxation timescale; see [5] for an example. Hence, for these problems, there may be considerable advantage in direct approximation of the global attractor, thus avoiding some of the long timescales associated with the transient behaviour. For gradient systems, this is equivalent to the approximation of heteroclinic orbits, provided that all equilibria are hyperbolic. Hale and Raugel [19] recently proved some results about the convergence of attractors for gradient systems under various approximation schemes.

Numerical methods for heteroclinic and homoclinic connections in ordinary differential equations (ODEs) are considered by Beyn in [1] and [2], and also by Doedel and Friedman in [14] and [10]. The basis of the approach in [1] is to consider the connecting orbit problem as a boundary value problem (BVP) on an infinite time domain, which is then approximated by appropriate truncation to a finite time domain. The truncation is determined by projecting the solution onto the stable manifold of the $\omega$ limit set and onto the unstable manifold of the $\alpha$ limit set. Beyn's approach was designed specifically for systems in which heteroclinic orbits occur for certain isolated parameter values.

In gradient systems, heteroclinic orbits do not occur at isolated parameter values but are generic for all parameter values. Furthermore, the connections often form a manifold of dimension greater than 1 . It is our aim to extend the method in [1] to cope with this situation and also to design methods effective for the large systems that arise from discretization of PDEs. Initially, we concentrate on a scalar reaction-diffusion equation (a generalisation of the Chafee-Infante problem) for which the geometric theory has been studied in detail [20], [21], [17]. This enables us to validate our method against theoretical properties of the connections. Our numerical techniques are easily generalized to other gradient systems, and we present numerical results for heteroclinic connections in the Cahn-Hilliard equation. In fact, we introduce a one-parameter family of PDEs connecting the Chafee-Infante and Cahn-Hilliard equations, and we demonstrate numerically that the attractor for the first of these equations can be continuously deformed into that of the second.

The plan of this paper is as follows. In $\S 2$ we describe the scalar reaction-diffusion equation, which we use as a model to develop our techniques. We describe various known results about the dynamics of the problem (Theorems 2.1, 2.2, 2.9, and 2.10) and, in addition, describe some new results about the Fourier spanning bases of the equilibrium solutions and certain heteroclinic connections (Theorems 2.6 and 2.7 and Proposition 2.8). In $\S 3$ we discretize the reaction diffusion equation by a Galerkin spectral method to obtain a system of ODEs for which we prove discrete analogues of the results for the underlying PDE. In particular, we establish that the system of ODEs forms a dissipative dynamical system with a gradient structure (Theorems 3.2 and 3.3), and we also show that the results about Fourier spanning bases have discrete counterparts. In $\S 4$ we extend the method of [1] to the system of ODEs. We introduce a continuation technique to study families of connections on the global 
attractor; starting points are found for this continuation technique by restricting the equation to certain invariant subspaces that result from the Fourier spanning bases. In these invariant subspaces, heteroclinic connections may be computed as solutions of initial value problems (see Remark 3.5). In $\S 5$ we present numerical results for the Chafee-Infante problem [6], and in $\S 6$ for the Cahn-Hilliard equation [4], [12].

2. Properties of the reaction-diffusion equation. In this section, we consider the PDE

$$
\begin{aligned}
& \frac{\partial u}{\partial t}=\frac{\partial^{2} u}{\partial x^{2}}-\lambda f(u), \quad x \in(0,1), \quad t>0, \\
& u(0, t)=u(1, t)=0, \quad t>0, \\
& u(x, 0)=u_{0}(x), \quad x \in[0,1],
\end{aligned}
$$

where it is assumed that

$$
f(s)=\sum_{j=0}^{p} b_{j} s^{2 j+1}, \quad b_{p}>0, \quad b_{0}=-1 .
$$

Consequently, $f$ satisfies the following properties:

(F1) $f \in C^{\infty}(R, R)$;

(F2) There exists $\bar{u}$ such that $f(u) / u>0$, for any $u$ with $|u|>\bar{u}$;

(F3) There exist $a, b>0$ such that $f^{\prime}(u) \geq a u^{2 p}-b$ for any $u \in R$.

The canonical example is the Chafee-Infante equation [6], where $f(u)=\left(u^{3}-u\right)$.

In the following, we use $L^{p}$ and $H_{0}^{1}$ to denote the Sobolev spaces $L^{p}(0,1)$ and $H_{0}^{1}(0,1)$, and the $L^{\infty}, L^{2}$, and $H_{0}^{1}$ norms are denoted by

$$
|u|_{\infty}=\sup _{x \in(0,1)}|u(x)|, \quad|u|^{2}=\int_{0}^{1} u^{2}(x) d x, \quad\|u\|^{2}=\int_{0}^{1} u_{x}^{2}(x) d x
$$

respectively.

Equations (2.1)-(2.4) have the following important properties, which we wish to capture in any numerical approximation. The results may be found in [17] and [26].

THEOREM 2.1. We have the following conditions:

(P1) Given $u_{0} \in L^{2}$, there exists a unique solution $u$ of (2.1)-(2.4) that satisfies

$$
\begin{gathered}
u \in L^{2}\left(0, T ; H_{0}^{1}\right) \cap L^{2 p+2}\left(0, T ; L^{2 p+2}\right) \quad \text { for any } T>0, \\
u \in C\left(R_{+} ; L^{2}\right) .
\end{gathered}
$$

The mapping $u_{0} \rightarrow u(t)$ is continuous in $L^{2}$, so that a semigroup $S(t)$ may be defined as

$$
S(t): u_{0} \in L^{2} \rightarrow u(t) \in L^{2}
$$

(P2) Given $u_{0} \in L^{2}$, there exist absorbing sets in $L^{2}$ and $H_{0}^{1}$. That is, there exist constants $\rho_{0}, \rho_{1}$, and times $t_{0}, t_{1}$ depending only on $\rho_{0}, \rho_{1}$, such that

$$
\begin{array}{ll}
|u| \leq \rho_{0} & \forall t \geq t_{0} \\
\|u\| \leq \rho_{1} & \forall t \geq t_{1}
\end{array}
$$


(P3) The semigroup $S(t)$ possesses a maximal attractor $\mathcal{A}$ that is bounded in $H_{0}^{1}$ and compact and connected in $L^{2}$;

(P4) Equations (2.1)-(2.4) have a gradient structure. Specifically, the functional

$$
I(v):=\int_{0}^{1}\left(\frac{v_{x}^{2}}{2}+\lambda F(v)\right) d x
$$

where

$$
F^{\prime}(v)=f(v)
$$

is a Lyapunov functional satisfying (i) $I(v) \geq \frac{1}{4}\|v\|^{2}-C_{0}$; (ii) $\frac{d}{d t}[I(u(t))]=-\left|u_{t}\right|^{2}$, $t>0$; and (iii) $I(S(t) v)=I(v)$ for $t \in R^{+}$implies that $v$ is contained in the set $\mathcal{E}$ of equilibrium points of (2.1)-(2.4);

$(\mathrm{P} 5)$ Let $W^{u}(v)$ denote the unstable manifold of an equilibrium $v \in \mathcal{E}$. If each member of $\mathcal{E}$ is hyperbolic, then

$$
\mathcal{A}=\bigcup_{v \in \mathcal{E}} W^{u}(v)
$$

We now discuss the properties of the set of equilibria $\mathcal{E}$. By an equilibrium point, we mean a twice continuously differentiable function $v$ satisfying

$$
\begin{gathered}
-v_{x x}+\lambda f(v)=0, \\
v(0)=v(1)=0 .
\end{gathered}
$$

THEOREM 2.2. For (2.1)-(2.4), $\mathcal{E}$ is bounded in the $H_{0}^{1}$ and supremum norms. Specifically, for any $v \in \mathcal{E}$,

$$
|v(x)|_{\infty} \leq \bar{u}, \quad\|v(x)\|^{2} \leq C_{1},
$$

where

$$
C_{1}=\max _{|u| \leq \bar{u}}[-\lambda u f(u)]
$$

Proof. Assume that $v(x)$ attains a maximum at a point $x^{*} \in(0,1)$ with $v\left(x^{*}\right)>\bar{u}$. By $(\mathrm{F} 2), f\left(v\left(x^{*}\right)\right)>0$, and, since the point is a maximum, $v_{x x}\left(x^{*}\right) \leq 0$. This contradicts (2.6), and the supremum bound follows. To establish the $H_{0}^{1}$ bound, take the inner product of (2.6) with $v$ to obtain, by (F2) and (2.8),

$$
\|v\|^{2}=-\langle\lambda f(v), v\rangle \leq C_{1} .
$$

Now we examine the invariance properties of problem $(2.6),(2.7)$. In particular, we determine Fourier spanning bases for branches of steady solutions. The analysis is then extended to the time-dependent problem (2.1)-(2.3). The basis of the results is to show that certain Fourier bases are invariant under $f$. The following result is well known and required for the bifurcation analysis.

LEMma 2.3. The spectrum $\sigma(A)$ of the operator $A: C_{0}^{2}[0,1] \rightarrow C_{0}[0,1]$ defined by $A \phi=-\phi^{\prime \prime}(x)$ consists of simple eigenvalues

$$
\left\{\pi^{2}, 4 \pi^{2}, \ldots, n^{2} \pi^{2}, \ldots\right\}
$$


with the following as their corresponding eigenfunctions

$$
\{\sin (\pi x), \sin (2 \pi x), \ldots, \sin (n \pi x), \ldots\}
$$

It is well known that the space of two-periodic, odd, continuous functions is spanned by (2.9). Thus it is natural to commence the study of $(2.6),(2.7)$ by introducing the spaces

$$
\begin{gathered}
\mathbf{X}:=C^{2} \cap \operatorname{span}\{\sin (j \pi x) \mid j \in \mathbf{N}\} \\
\mathbf{Y}:=C \cap \operatorname{span}\{\sin (j \pi x) \mid j \in \mathbf{N}\}
\end{gathered}
$$

where $\mathbf{N}$ is the set of natural numbers. Clearly, $\mathbf{X} \subset \mathbf{Y}$. (Note that the Dirichlet boundary conditions (2.7) are automatically satisfied by functions in $\mathbf{X}$ and $\mathbf{Y}$.) For reasons that will become clear in Lemma 2.5, we consider those functions in $\mathbf{X}$ that have the additional property that they are even about $\frac{1}{2} k,(k \in \mathbf{N})$, i.e.,

$$
u(x)=u(1 / k-x), \quad k \in \mathbf{N} .
$$

Odd continuous functions satisfying (2.10) have particularly simple Fourier bases.

LEMmA 2.4. Assume that $u$ is odd, continuous, and satisfies (2.10). Then, for given $k \in \mathbf{N}$, (i) $u$ is odd about $1 / k$; (ii) $u$ has period $2 / k$ (and hence has period 2 ); and, (iii) $u \in \operatorname{span}\{\sin ((2 j-1) k \pi x) \mid j \in \mathbf{N}\}$.

Proof. (i) By the definition, it is clear that

$$
u(1 / k-x)=u(x)=-u(-x)=-u(1 / k-(-x))=-u(1 / k+x) .
$$

(ii) Since

$$
\begin{aligned}
u(x)=u(1 / k-x)=-u(1 / k+x) & =u(-1 / k-x) \\
& =u(1 / k-(-1 / k-x))=u(2 / k+x),
\end{aligned}
$$

then

$$
u(x)=u\left(x+\frac{2 m}{k}\right), \quad m \in \mathbf{N},
$$

and taking $m=k$ gives $u(x)=u(x+2)$.

(iii) Consider the Fourier series of $u$ over $(-1 / k, 1 / k)$, since $u$ has period $2 / k$. Since $u$ is odd about zero, then

$$
a_{n}=\frac{2}{1 / k} \int_{-1 / k}^{1 / k} u(x) \cos \frac{n \pi x}{1 / k} d x=0, \quad n=1,2, \ldots,
$$

and

$$
b_{n}=\frac{2}{1 / k} \int_{0}^{1 / k} u(x) \sin \frac{n \pi x}{1 / k} d x=2 k \int_{0}^{1 / k} u(x) \sin (n k \pi x) d x, \quad n=1,2, \ldots
$$

For $n$ even, say $n=2 m$, we have

$$
\sin (2 m k \pi(1 / k-x))=\sin (2 m \pi-2 m k \pi x)=-\sin (2 m k \pi x) .
$$


Hence $\sin (n k \pi x)$ is odd about $\frac{1}{2} k$. However, $u(x)$ is even about $\frac{1}{2} k$. Thus $b_{n}=0$ for $n$ even, so that the Fourier series for $u$ over $(-1 / k, 1 / k)$ contains only terms of the form

$$
\sin ((2 j-1) k \pi x), \quad j \in \mathbf{N} \text {. }
$$

By periodicity, the Fourier series of $u$ over $(0,1)$ and $(-1,1)$ also contains only these terms.

Thus, for $k \in \mathbf{N}$, we may introduce the subspaces $\mathbf{X}_{k}, \mathbf{Y}_{k}$ of $\mathbf{X}, \mathbf{Y}$, which are given by

$$
\begin{gathered}
\mathbf{X}_{k}=C^{2} \cap \operatorname{span}\{\sin ((2 j-1) k \pi x), j \in \mathbf{N}\}, \\
\mathbf{Y}_{k}=C \cap \operatorname{span}\{\sin ((2 j-1) k \pi x), j \in \mathbf{N}\}, \quad k=1,2, \ldots
\end{gathered}
$$

Clearly, $\mathbf{X}_{k} \subset \mathbf{Y}_{k}$. Functions in $\mathbf{Y}_{k}$ are odd, $2 / k$ periodic, even about $\frac{1}{2} k$, and satisfy (2.7). We introduce the operator $H(v, \lambda): \mathbf{X} \times R \rightarrow \mathbf{Y}$ defined by

$$
H(v, \lambda):=v_{x x}-\lambda f(v)
$$

where $f(u)$ is given by $(2.4)$. The following "invariance" lemma makes clear the reason for the introduction of property (2.10) and the subspaces $\mathbf{X}_{k}, \mathbf{Y}_{k}$.

Lemma 2.5. Suppose that $H$ is defined as (2.11); then (a) $f: \mathbf{X}_{k} \rightarrow \mathbf{X}_{k}$; (b) $H(v, \lambda): \mathbf{X}_{k} \times R \rightarrow \mathbf{Y}_{k}$.

Proof. It holds that (b) follows if (a) is true. The proof of (a) follows from the fact that, if $u$ is odd, continuous, and satisfies (2.10), then any odd power of $u$ is also odd, continuous, and satisfies (2.10). Hence, by the Lemma 2.4, any odd power of $u$ maps $\mathbf{X}_{k}$ into itself. So $f$, which is defined by (2.4), also does.

The bifurcation analysis of $H(v, \lambda)$ (and hence (2.6), (2.7)) can now be carried out in the subspaces $\mathbf{X}_{k}, \mathbf{Y}_{k}$. Clearly, $H(0, \lambda)=0$. The solution of $H_{v}(0, \lambda) \phi=0$ reduces to finding $\phi$, shown below:

$$
\phi^{\prime \prime}+\lambda \phi=0, \quad \phi \in \mathbf{X}_{k}
$$

By Lemma 2.3, we know that the only possible bifurcation points giving rise to solutions in $\mathbf{X}_{k}$ are at $\lambda_{k(2 j-1)}=k^{2}(2 j-1)^{2} \pi^{2}, j \in \mathbf{N}$ where the one-dimensional null spaces are spanned by $\phi_{k(2 j-1)}=\sin (2 j-1) k \pi x, k=1,2, \ldots$ Since, for $\phi=\phi_{k(2 j-1)}$ and $\lambda=\lambda_{k(2 j-1)}, H_{v \lambda}(0, \lambda) \phi=-\phi$, the nondegeneracy condition

$$
H_{v \lambda}(0, \lambda) \phi \notin \text { Range }\left\{H_{v}(0, \lambda)\right\}
$$

holds at the bifurcation points, and so bifurcation does occur (see, for example, Chow and Hale [8]). In addition, if we introduce the reflectional symmetry $\sigma$, defined on $\mathbf{X}$ and $\mathbf{Y}$ by

$$
\sigma v(x)=-v(x), \quad \sigma^{2}=I, \quad \sigma \neq I,
$$

then clearly $H$ satisfies the equivariance condition

$$
H(\sigma v, \lambda)=\sigma H(v, \lambda), \quad v \in X
$$

The symmetric solutions $(\sigma v=v)$ are the trivial solution, and so the bifurcations at $\left(0, \lambda_{k(2 j-1)}\right)$ are symmetry-breaking and hence pitchfork. Thus we have proved the following theorem. 
TheOREM 2.6. For any $k \in \mathbf{N}$, consider $H(v, \lambda): \mathbf{X}_{k} \times R \rightarrow \mathbf{Y}_{k}$ as defined by (2.11). The points $\left(0, \lambda_{k(2 j-1)}\right), j \in \mathbf{N}$ are symmetry-breaking pitchfork bifurcation points on the trivial solution branch. Hence there are two bifurcating branches in $\mathbf{X}_{k} \backslash\{0\}$ that are conjugate in the sense that, if $(v, \lambda)$ is a solution of $H(v, \lambda)=0$, then so is $(-v, \lambda)$. Finally, if $b_{1} \neq 0$ in $(2.4)$, then

$$
H_{v v v} \phi_{k(2 j-1)} \phi_{k(2 j-1)} \phi_{k(2 j-1)} \notin \text { Range }\left\{H_{v}\left(0, \lambda_{k(2 j-1)}\right)\right\} \text {, }
$$

and the bifurcation is quadratic pitchfork.

If we now consider the bifurcation problem for $H(v, \lambda)=0$ in the whole space, that is, consider $H(v, \lambda): \mathbf{X} \times R \rightarrow \mathbf{Y}$, then it is natural to ask if any additional solutions arise that are not in $\mathbf{X}_{k}$ for any $k \in \mathbf{N}$. However, $\left([k(2 j-1)]^{2} \pi^{2}, \phi_{k(2 j-1)}\right)$ is a simple eigenpair of $H_{v}(0, \lambda) \phi=0$ in $\mathbf{X}$, and so the pitchfork bifurcation gives rise to a unique pair of conjugate solutions in $\mathbf{X}$ as well as in $\mathbf{X}_{k}$. Furthermore, $\left\{[k(2 j-1)]^{2} \pi^{2}\right\}_{k, j \in \mathbf{N}}=\left\{\pi^{2}, 4 \pi^{2}, 9 \pi^{2}, \cdots\right\}$, and so, by varying $k \in \mathbf{N}$, Theorem 2.6 covers all the possible bifurcation points in $\mathbf{X}$.

It is possible now to consider $(2.1)-(2.3)$ as a differential equation defined on appropriate spaces that admit only steady solutions in $\mathbf{X}_{k}$. In [17] it is proved that (2.1)-(2.3) defines a dynamical system on $H_{0}^{1}$. A straightforward modification of that result shows that the evolution equation (2.1)-(2.3) also defines a dynamical system on $\mathbf{Z}_{k}$ defined by

$$
\mathbf{Z}_{k}:=H_{0}^{1} \cap \operatorname{span}\{\sin ((2 j-1) k \pi x) \mid j \in \mathbf{N}\}
$$

where $\mathbf{N}$ is the set of natural numbers. This gives the following theorem.

THEOREM 2.7. Given $u_{0} \in \mathbf{Z}_{k}$ there exists a unique solution $u$ of $(2.1)-(2.3)$ with $u \in \mathbf{Z}_{k}$. The mapping $u_{0} \rightarrow u(t)$ is $C^{1}$ on $\mathbf{Z}_{k}$ so that a $C^{1}$ semigroup $S(t)$ may be defined as

$$
S(t): u_{0} \in \mathbf{Z}_{k} \rightarrow u(t) \in \mathbf{Z}_{k}
$$

It is important to realize that the steady and time-dependent solutions predicted by Theorems 2.6 and 2.7 follow from the invariance of the space

$$
\mathbf{S}_{k}:=\operatorname{span}\{\sin ((2 j-1) k \pi x), j \in \mathbf{N}\}
$$

under $f(u)$. As such, their existence can be predicted by a simple rescaling argument: consider any solution $u(x, t)$ of $(2.1)-(2.4)$ with $u(x, t) \in \mathbf{S}_{1}$ for each $t \geq 0$. Extend this function from a solution defined on $[0,1]$ to a solution defined on $[0, k]$, for any integer $k \geq 2$, by odd reflection of unit intervals about $x=1,2, \ldots, k-1$. This function can then be mapped back onto [0,1], and satisfies the original problem with $\lambda \rightarrow \lambda k^{2}$ and $t \rightarrow k^{-2} t$. The newly constructed solution lies in $\mathbf{S}_{k}$.

This argument suggests the following proposition, which is useful for the construction of starting points for the computation of heteroclinic connections that are dynamically unstable.

Proposition 2.8. If, for given $\lambda$, a steady solution $u(x ; \lambda) \in \mathbf{X}_{1}$ is stable (in a given topology) as a solution of (2.1)-(2.4), then the solution $u\left(x / k ; k^{2} \lambda\right) \in \mathbf{X}_{k}$, found by the extension described above, is stable (in the same topology) as a solution of (2.1)-(2.4) restricted to initial data $u_{0} \in \mathbf{Z}_{k}$.

We now describe in detail the structure of solutions to the generalized ChafeeInfante problem defined by $(2.1)-(2.4)$ with the additional structural assumption, as follows: 


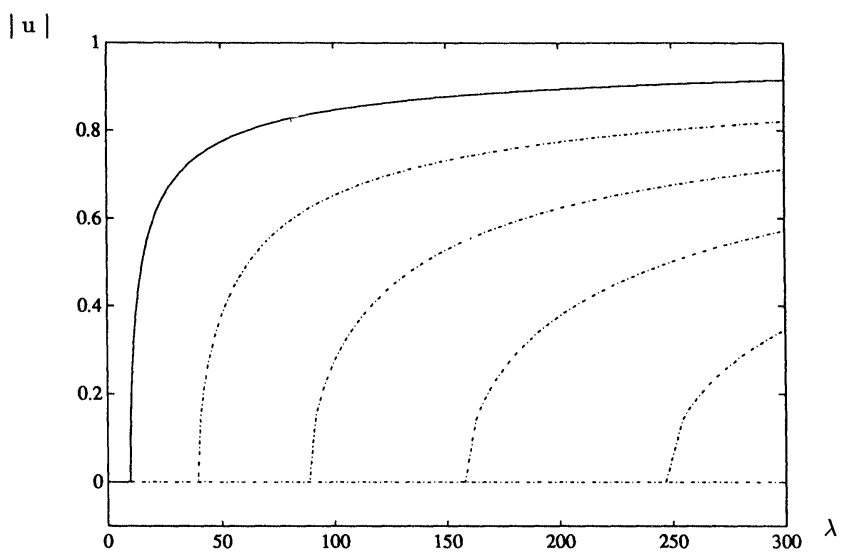

FIG. 2.1. Steady-state solutions of the Chafee-Infante problem. The solid line (-) denotes stable branches, and the dashed line (-...) denotes unstable branches.

(F4) $u f^{\prime \prime}(u)>0$ for all $u \neq 0$.

This example is used as both an illustration and as a test of our numerical methods, so it is important to understand its solution behaviour. The structure and stability of the set of equilibria $\mathcal{E}$ is captured in the following theorem from [6]. In this theorem, stability is defined in terms of the usual supremum norm for $C^{1}([0,1])$ functions.

THEOREM 2.9. Assume that $f(u)$ defined by (2.4) satisfies $(\mathrm{F} 4)$. For each integer $n \geq 1$, let $\lambda_{n}=n^{2} \pi^{2}$. Then, for any $n \geq 1$ and $\lambda \in\left[\lambda_{n},+\infty\right),(2.1)-(2.4)$ have two equilibrium points $u_{n}^{ \pm}(\lambda)$ such that (i) $u_{n}^{ \pm}\left(\lambda_{n}\right)=0$;

(ii) For any $\lambda \in\left(\lambda_{n},+\infty\right), u_{n}^{ \pm}(\lambda)$ has exactly $n+1$ zeros in $[0,1]$. Furthermore, for any $\lambda \in[0,+\infty),(2.1),(2.2)$ have no equilibrium points other than $u \equiv 0$ and those elements $u_{n}^{ \pm}(\lambda), n \geq 1$, for which $\lambda_{n} \leq \lambda$;

(iii) For each $\lambda \in\left[0, \bar{\lambda}_{1}\right]$, the solution $u \equiv 0$ is asymptotically stable, and for each $\lambda \in\left(\lambda_{1},+\infty\right) u \equiv 0$ is unstable;

(iv) For each $n \geq 1$ and any $\lambda \in\left(\lambda_{1},+\infty\right)$, the equilibrium point $u_{1}^{ \pm}(\lambda)$ is asymptotically stable, and, for any $\lambda \in\left[\lambda_{n},+\infty\right), n>1$, the equilibrium point $u_{n}^{ \pm}(\lambda)$ is unstable.

Note that, by Theorem 2.6, the branch of solutions $u_{k(2 j-1)}^{ \pm}(\lambda)$ for some pair $k, j \in \mathbf{N}$ lies in $\mathbf{X}_{k}$. Figure 2.1 shows the steady solutions in the case where $f(u)=$ $u^{3}-u$, and the appropriate spanning bases are marked.

We now describe the structure of heteroclinic orbits; see [21] and [17] for details. Define the stable and unstable manifolds of an equilibrium $\phi$ of $(2.1),(2.2)$ as follows, where $H_{0}^{r}:=H_{0}^{r}(0,1)$ for some $\frac{3}{2}<r<2$ :

$$
\begin{gathered}
W^{s}(\phi)=\left\{u_{0} \in H_{0}^{r} \mid S(t) u_{0} \text { exists for all } t \geq 0 \text { and tends to } \phi \text { as } t \rightarrow+\infty\right\} \\
W^{u}(\phi)=\left\{u_{0} \in H_{0}^{r} \mid \text { there is a solution } S(t) u_{0} \text { of }(2.1),(2.2)\right. \text { on } \\
\left.\quad-\infty<t \leq 0, S(t) u_{0} \text {, and } S(t) u_{0} \text { tends to } \phi \text { as } t \rightarrow-\infty\right\}
\end{gathered}
$$

Consider any equilibria $\phi$ and $\psi$ of $(2.1),(2.2)$; if the set $W^{u}(\phi) \bigcap W^{s}(\psi)$ is not empty, then it contains all connections from the equilibrium $\phi$ at $-\infty$ to $\psi$ at $+\infty$. It is possible to determine whether a connection exists and to determine its dimension by studying the $\operatorname{index}(\phi)$ of steady states, which is defined to be the number of positive 
eigenvalues of the Frechet derivative of $H$ defined by (2.11) at $\phi \in \mathcal{E}$. The following result is proved in [21].

THEOREM 2.10. Consider (2.1)-(2.4) and, in addition, assume that $f(u)$ satisfies (F4). Let $\lambda \geq 0, \lambda \notin\left\{\pi^{2}, 4 \pi^{2}, 9 \pi^{2}, \ldots\right\}$ and let $\phi, \psi$ be any two equilibria. Then

$$
W^{u}(\phi) \bigcap W^{s}(\psi) \neq \text { empty }
$$

(there exist connections from $\phi$ to $\psi$ ) if and only if either $\phi=\psi$ (in which case, the trivial connection is the equilibrium $\phi$ itself) or $\operatorname{ind} \operatorname{dex}(\phi)>\operatorname{index}(\psi)$. Furthermore,

$$
\operatorname{dim}\left[W^{u}(\phi) \bigcap W^{s}(\psi)\right]=\operatorname{index}(\phi)-\operatorname{index}(\psi) .
$$

Specifically, for any $n^{2} \pi^{2}<\lambda<(n+1)^{2} \pi^{2}$, the equilibria of the Chafee-Infante equation are denoted by $u_{1}^{ \pm}, u_{2}^{ \pm}, \ldots, u_{n}^{ \pm}$, and zero, which are the first, second,..., $n$ th, and trivial steady-state branches, respectively. Then

$$
\operatorname{index}(0)=n, \quad i \operatorname{index}\left(u_{i}^{ \pm}\right)=i-1, \quad(i=1,2, \ldots, n) .
$$

Hence the dimension of the set of connections between any pair of solutions $u_{i}$ and $u_{j}$ can be explicitly written as

$$
\begin{gathered}
\operatorname{dim}\left[W^{u}\left(u_{i}^{ \pm}\right) \bigcap W^{s}\left(u_{j}^{ \pm}\right)\right]=i-j, \quad(i>j), \\
\operatorname{dim}\left[W^{u}(0) \bigcap W^{s}\left(u_{i}^{ \pm}\right)\right]=n-i+1, \quad(1 \leq i \leq n) .
\end{gathered}
$$

3. Properties of the semi-discrete system. In this section, we describe the Galerkin spectral method, which we use to derive a finite-dimensional system of ODEs from the PDE (2.1)-(2.4). This system of ODEs is then used as the basis for our numerical computation of connecting orbits. For this reason, it is important to show that the system of ODEs inherits the underlying structure of the PDE. First, we prove that Theorems 2.1 and 2.2 have appropriate discrete analogues for the spectral method, giving a sound theoretical underpinning to our computational technique. Second, we note that the theorems on the Fourier spanning basis properties of (2.1)(2.4) have appropriate discrete analogues, and this is then exploited in $\S 4$ to construct an efficient numerical algorithm.

We seek an approximation to (2.1)-(2.4) by means of a Galerkin spectral method, using the eigenfunctions $\{\sin (j \pi x)\}_{j=1}^{\infty}$ as a basis. Specifically, we set

$$
u_{\mathrm{N}}(x, t)=\sum_{j=1}^{N} a_{j}(t) \sin (j \pi x)
$$

Let $A=\left(a_{1}(t), a_{2}(t), \cdots, a_{\mathrm{N}}(t)\right)^{T} \in R^{N}$. We introduce the norms

$$
|A|_{0}^{2}=\frac{1}{2} \sum_{k=1}^{N} a_{k}^{2}, \quad|A|_{1}^{2}=\frac{1}{2} \sum_{k=1}^{N} k^{2} \pi^{2} a_{k}^{2}, \quad|A|_{\infty}=\max _{k}\left|a_{k}\right|
$$

to denote discrete $L^{2}$ and $H_{0}^{1}$ norms and the standard maximum norm in $R^{N}$. Note that

$$
\begin{gathered}
\frac{|A|_{\infty}}{\sqrt{2}} \leq|A|_{0} \leq \frac{|A|_{1}}{\pi}, \\
|A|_{1} \leq N \pi|A|_{0} .
\end{gathered}
$$


We denote the normed vector space $\left(R^{N},|\bullet|_{0}\right)$ by $L_{N}^{2}$.

Applying the Galerkin spectral technique gives

$$
\left\langle\frac{\partial u_{\mathrm{N}}}{\partial t}-\frac{\partial^{2} u_{\mathrm{N}}}{\partial x^{2}}+\lambda f\left(u_{\mathrm{N}}\right), \quad \sin (k \pi x)\right\rangle=0, \quad k=1,2, \ldots, N,
$$

where $\langle\bullet, \bullet\rangle$ denotes the usual $L^{2}$ inner product on $[0,1]$, defined by

$$
\langle f(x), g(x)\rangle=\int_{0}^{1} f(x) g(x) d x .
$$

This then yields the following system of ODEs:

$$
\begin{gathered}
\frac{d a_{k}(t)}{d t}=-a_{k}(t) \pi^{2} k^{2}-2 \lambda \int_{0}^{1} f\left(u_{\mathrm{N}}(x, t)\right) \sin (k \pi x) d x, \\
a_{k}(0)=a_{k}^{0}=\int_{0}^{1} u_{0}(x) \sin (k \pi x) d x, \quad k=1,2, \ldots, N .
\end{gathered}
$$

We formally rewrite the system $(3.4),(3.5)$ as

$$
\frac{d A(t)}{d t}=G(A(t), \lambda), \quad A(0)=A_{0}
$$

where

$$
A_{0}=\left(a_{1}^{0}, a_{2}^{0}, \ldots, a_{\mathrm{N}}^{0}\right)^{T}
$$

The following a priori estimates are needed.

Lemma 3.1. Solutions $A(t)$ of (3.4) satisfy

$$
\begin{array}{ll}
\text { (i) } & \frac{1}{2} \frac{d}{d t}|A|_{0}^{2}+|A|_{1}^{2} \leq C_{1}, \\
\text { (ii) } & \frac{1}{2} \frac{d}{d t}|A|_{1}^{2}+\left\langle\lambda f^{\prime}\left(u_{\mathrm{N}}\right)\left(u_{\mathrm{N}}\right)_{x},\left(u_{\mathrm{N}}\right)_{x}\right\rangle \leq 0, \\
\text { (iii) } & \frac{d}{d t} I^{N}(A)=-\left|\frac{d A}{d t}\right|_{0}^{2},
\end{array}
$$

where

$$
I^{N}(A)=\frac{1}{2}|A|_{1}^{2}+\left\langle\lambda F\left(u_{\mathrm{N}}\right), 1\right\rangle
$$

and $F$ is defined by (2.5).

Proof. (i) Multiplying (3.4) by $a_{k}$, summing, and using (2.8) gives

$$
\frac{1}{2} \frac{d}{d t}|A|_{0}^{2}+|A|_{1}^{2} \leq-\lambda \int_{0}^{1} f\left(u_{\mathrm{N}}(x, t)\right) u_{\mathrm{N}}(x, t) d x \leq C_{1} .
$$

(ii) Multiplying (3.4) by $k^{2} \pi^{2} a_{k}$, summing, and integrating by parts gives the result.

(iii) Multiplying (3.4) by $d a_{k} / d t$ and summing gives the result.

We now prove a discrete analogue of Theorem 2.1. Similar results may be found for fully discrete Galerkin methods in Shen [25] and for fully discrete finite difference methods in Elliott and Stuart [13]. Note that for result (PD2), below, the absorbing 
set in the $|\bullet|_{1}$ norm is $\mathrm{N}$-independent, although no such uniform bound is assumed on the $|\bullet|{ }_{1}$ norm of the initial data - in this sense, (PD2) generalizes (P2).

THEOREM 3.2. We have the following conditions:

(PD1) Given $A_{0} \in L_{N}^{2}$, there exists a unique solution $A$ of $(3.4),(3.5)$, which satisfies

$$
A \in C^{1}\left(0, T ; L_{N}^{2}\right) \quad \forall T>0 .
$$

The mapping $A_{0} \rightarrow A(t)$ is continuous in $L_{N}^{2}$ so that a continuous semigroup $S^{N}(t)$ may be defined by

$$
S^{N}(t): A_{0} \in L_{N}^{2} \rightarrow A(t) \in L_{N}^{2}
$$

(PD2) Given $A_{0} \in L_{N}^{2}$, then there exist absorbing sets in the $|\bullet|_{0}$ and $|\bullet|_{1}$ norms. That is, there exist constants $\rho_{0}, \rho_{1}$, and times $t_{0}, t_{1}$ independent of $N$, and depending only on $A_{0}, \rho_{0}$, and $\rho_{1}$ such that

$$
\begin{aligned}
& |A(t)|_{0}^{2} \leq \rho_{0} \quad \forall t \geq t_{0}, \\
& |A(t)|_{1}^{2} \leq \rho_{1} \quad \forall t \geq t_{1}
\end{aligned}
$$

(PD3) The semigroup $S^{N}(t)$ possesses a maximal attractor $\mathcal{A}^{N}$, which is bounded independent of $N$ in the $|\bullet|_{1}$ norm and compact and connected in $L_{N}^{2}$;

(PD4) Equations (3.4), (3.5) have a gradient structure. Specifically, the functional $I^{N}(A(t))$ defined by (3.10) satisfies (i) $I^{N}(A) \geq \frac{1}{4}\|A\|_{1}^{2}-C_{2}$; (ii) $d / d t\left[I^{N}(A(t))\right]=$ - $|d A(t) / d t|_{0}^{2}, t>0$; (iii) $I^{N}\left(S^{N}(t) A\right)=I^{N}(A)$ for $t \geq 0$ implies that $A$ is contained in the set $\mathcal{E}^{N}$ of equilibrium points of (3.4).

(PD5) Let $W^{u}(A)$ denote the unstable manifold of an equilibrium $A \in \mathcal{E}^{N}$. If each member of $\mathcal{E}^{N}$ is hyperbolic, then

$$
\mathcal{A}^{N}=\bigcup_{A \in \mathcal{E}^{N}} W^{u}(A) .
$$

Proof. (PD1) Local existence and uniqueness follows from the standard theory of ODEs, since $f \in C^{\infty}(R, R)$ by (F1). We now establish an a priori bound on $|A|_{\infty}$. By (3.7), (3.2), we have

$$
\frac{1}{2} \frac{d}{d t}|A|_{0}^{2}+\pi^{2}|A|_{0}^{2} \leq C_{1}
$$

Integrating, we obtain

$$
|A(t)|_{0}^{2} \leq e^{-2 \pi t}\left|A_{0}\right|_{0}^{2}+\frac{C_{1}}{\pi^{2}}\left[1-e^{-2 \pi t}\right]
$$

Thus $|A(t)|_{0}^{2}$ is uniformly bounded for all $t>0$. Hence, by (3.2), the same is true for $|A(t)|_{\infty}$. Global existence and uniqueness follows from this bound. Continuity with respect to initial data also follows directly.

(PD2) The first result follows from (3.13), with any $\rho_{0}>C_{1} / \pi^{2}$. To establish the second result, apply (F3) to (3.8) to give

$$
\frac{1}{2} \frac{d}{d t}|A(t)|_{1}^{2} \leq \int_{0}^{1}\left[b-a\left(u_{\mathrm{N}}\right)^{2 p}\right]\left(u_{\mathrm{N}}\right)_{x}^{2} d x \leq b|A|_{1}^{2} .
$$


Now, by (3.7),

$$
\frac{1}{2}|A(t+\theta)|_{0}^{2}-\frac{1}{2}|A(t)|_{0}^{2}+\int_{t}^{t+\theta}|A(t)|_{1}^{2} d t \leq C_{1} \theta
$$

thus

$$
\int_{t}^{t+\theta}|A(t)|_{1}^{2} d t \leq C_{1} \theta+\frac{1}{2} \rho_{0} \quad \forall t \geq t_{0}
$$

Thus, applying the uniform Gronwall lemma [26] to (3.14), we obtain for $t$ sufficiently large,

$$
|A(t+\theta)|_{1}^{2} \leq\left(C_{1}+\frac{\rho_{0}}{2 \theta}\right) e^{2 b \theta} .
$$

Fixing $\theta>0$, we see that, for $t$ sufficiently large, we have an absorbing set in the $|\bullet|_{1}$ norm by setting

$$
\rho_{1}>\left(C_{1}+\frac{\rho_{0}}{2 \theta}\right) e^{2 b \theta}
$$

(PD3) From the absorbing set property (PD2), we deduce that the operators $S^{N}(t)$ are uniformly compact for sufficiently large $t$. Applying [26, Thm. 1.1, p. 23], we deduce the existence of a connected compact global attractor, defined by $\mathcal{A}=\omega\left(B_{i}\right)$ for either $i=0$ or 1 , where

$$
B_{i}:=\left\{A \in L_{N}^{2}:|A|_{i}^{2} \leq \rho_{i}\right\}
$$

The uniform bound in the $|\bullet|_{1}$ norm follows taking $i=1$.

(PD4) By $[17$, p. 76$]$, we deduce that

$$
F(u) \geq-\epsilon u^{2}-C_{\epsilon}
$$

for some $C_{\epsilon}>0$. Hence

$$
\left\langle F\left(u_{n}\right), 1\right\rangle \geq-\int_{0}^{1}\left[\epsilon U_{N}^{2}(x)+C_{\epsilon}\right] d x \geq-\epsilon|A|_{0}^{2}-C_{\epsilon} \geq-\frac{\epsilon}{\pi^{2}}|A|_{1}^{2}-C_{\epsilon} .
$$

Thus $I^{N}(A)$ defined by (3.10) satisfies

$$
I^{N}(A) \geq \frac{1}{4}\|A\|_{1}^{2}-C_{2},
$$

by choosing $\epsilon=\pi^{2} / 4 \lambda$. This establishes property (i). Property (ii) follows from (3.9). Finally, if $I^{N}\left(S^{N}(t) A\right)=I^{N}(A)$ for $t \geq 0$, then

$$
\frac{d I^{N}}{d t}\left(S^{N}(t) A\right)=0
$$

Hence, it holds that

$$
\left|\frac{d}{d t}\left(S^{N}(t) A\right)\right|_{0}^{2}=0
$$

by (3.9). Thus $A$ is an equilibrium point, and (iii) follows. 
(PD5) The system of equations is in gradient form. Furthermore, it is shown in Theorem 3.3 (below) that the set of equilibria $\mathcal{E}^{N}$ is bounded in the $|\bullet|_{1}$ norm and hence, by (3.2), in the supremum norm. Applying [17, Thm. 3.8.5] the result follows.

Let $\mathcal{E}^{N}$ denote the set of equilibria of (3.4). Then by (3.7) we trivially obtain the following theorem.

TheOREM 3.3. $\mathcal{E}^{N}$ is bounded in the $|\bullet|_{1}$ norm. Specifically, for any $A \in \mathcal{E}^{N}$,

$$
\text { | }\left.A\right|_{1} ^{2} \leq C_{1} \text {. }
$$

We now consider an algebraic property of the semidiscrete system, which is useful in the construction of the numerical methods for computing connections described in the next section.

Proposition 3.4. The steady-state solution of (3.6) is a system of nonlinear equations with real and symmetric Jacobian matrix.

Proof. We use the integral form of the steady state of (3.6), defined in (3.4). Thus

$$
G(A, \lambda)=\left(g_{1}(A, \lambda), g_{2}(A, \lambda), \ldots, g_{\mathrm{N}}(A, \lambda)\right)^{T},
$$

where

$$
g_{k}(A, \lambda)=-a_{k} \pi^{2} k^{2}+\lambda a_{k}-2 \lambda T_{k}, \quad k=1,2, \ldots, N,
$$

in which the $T_{k}$ is the nonlinear term given by

$$
T_{k}=T_{k}\left(a_{1}, a_{2}, \ldots, a_{\mathrm{N}}\right)=\int_{0}^{1} \sum_{m=1}^{p} b_{m}\left(\sum_{j=1}^{N} a_{j} \sin (j \pi x)\right)^{2 m+1} \sin (k \pi x) d x
$$

Hence the Jacobian matrix of (3.4) is real, and

$$
G_{A}(A, \lambda)=\Lambda-2 \lambda M,
$$

where

$$
\Lambda=\operatorname{diag}\left(\lambda-\pi^{2}, \lambda-2^{2} \pi^{2}, \lambda-3^{2} \pi^{2}, \ldots, \lambda-N^{2} \pi^{2}\right)
$$

and $M=\left(m_{i j}\right)_{\mathrm{N} \times \mathrm{N}}$. Here

$$
\begin{aligned}
& m_{i j}=\frac{\partial}{\partial a_{j}}\left(T_{i}\right) \\
&=\int_{0}^{1} \sum_{m=1}^{p} b_{m}(2 m+1)\left(\sum_{l=1}^{N} a_{l} \sin (l \pi x)\right)^{2 m} \sin (j \pi x) \sin (i \pi x) d x \\
& i, j=1,2, \ldots, N
\end{aligned}
$$

Clearly, $m_{i j}=m_{j i}$, and hence the matrix $M$ is symmetric. From (3.15), (3.16), it follows that $G_{A}(A, \lambda)$ is symmetric.

In $\S 2$ we showed that the differential equation (2.1)-(2.4) has invariant subspaces defined by intersection of standard function spaces with certain Fourier spanning bases. Because of the nature of the spectral method, these invariant subspaces have natural discrete analogues. It is possible to exploit this property in the numerical 
calculation of steady solutions and also in the computation of some connectionsspecifically, to compute the branch of steady solutions of (2.1)-(2.4) bifurcating from $\lambda_{k}=(k \pi)^{2}$ or a time-dependent solution to (2.1)-(2.4) with initial data in $\mathbf{Z}_{k}$ (see Theorems 2.6 and 2.7). To do this, we modify (3.1) and seek

$$
u_{\mathrm{N}}(x, t)=\sum_{j=1}^{N} a_{j}(t) \sin ((2 j-1) k \pi x),
$$

for given appropriate integer $k$. The use of this reduced spectral method is important for two main reasons.

Remark 3.5. (i) For steady solutions, the use of the Galerkin method based on (3.18) reduces computer time considerably since the dimension of the problems solved is far smaller.

(ii) For the computation of time-dependent problems, the use of (3.18) has the more important consequence that certain heteroclinic connections may be calculated purely by solving an initial value problem in an appropriate invariant subspace. We illustrate this by considering the Chafee-Infante problem. It is straightforward to calculate connections to stable limit sets at $t=+\infty$, since they can be found by solving initial value problems - whereas, general connections require the solution of boundary value problems (BVPs) in time. It is known (see Theorem 2.9) that for $\lambda>\pi^{2}$, the only stable solutions are $u_{1}^{ \pm}(\lambda)$, and hence that only heteroclinic connections with $u_{1}^{ \pm}(\lambda)$ as the limit set at $t=\infty$ will be stable and computable as solutions of an initial value problem. However, if we work with initial data in the subspace $\mathbf{Z}_{k}$, Proposition 2.8 shows that the branches of solutions $u_{k}^{ \pm}(\lambda)$ are stable. Hence, by working with a Galerkin method based on (3.18), we can compute certain connections that are unstable as a solution of (3.6) purely by solving an initial value problem. These form a starting point for the numerical continuation techniques described in the next section.

4. Numerical methods for connections. Heteroclinic connections for the reaction-diffusion problem $(2.1),(2.2)$ are given by solutions to the problem

$$
\begin{gathered}
\frac{\partial u}{\partial t}-\frac{\partial^{2} u}{\partial x^{2}}+\lambda f(u)=0, \quad x \in(0,1), \quad t \in(-\infty,+\infty), \\
u(0, t)=u(1, t)=0 \\
\lim _{t \rightarrow-\infty} u(x, t)=\phi \\
\lim _{t \rightarrow+\infty} u(x, t)=\psi
\end{gathered}
$$

where $\phi, \psi \in \mathcal{E}$ the set of equilibria. This is a BVP defined on an infinite interval. Note that the phase is free, and this must be set to obtain a well-posed BVP. Applying the Galerkin method, we obtain the following system of ODEs:

$$
\begin{gathered}
\frac{d A(t)}{d t}-G(A(t), \lambda)=0, \\
\lim _{t \rightarrow-\infty} A(t)=A_{-}, \quad \lim _{t \rightarrow+\infty} A(t)=A_{+},
\end{gathered}
$$

where $G: R^{N} \times R \rightarrow R^{N}$ is defined by (3.4)-(3.6), and $A_{-}$and $A_{+}$are steadystate solutions of system (4.1) belonging to $\mathcal{E}^{N}$ and are approximations to the steady solutions $\phi, \psi$. We solve problem $(4.1),(4.2)$ by truncating it to a problem that 
is defined on a finite interval, as in [1], [22], [24]. For the purpose of obtaining a well-defined BVP, we add a phase-fixing condition, and sometimes add what we call determining conditions. The determining conditions are needed when the dimension of the manifold of heteroclinic connections between two equilibria is greater than 1 (see Theorem 2.10); they are used to parameterize the family of connections so that numerical continuation may be used within the family.

Let the truncated interval be $\mathcal{T}=\left[T_{-}, T_{+}\right]$. Define a projection operator $L_{+u} \in$ $R^{N_{u} \times N}$ (respectively, $L_{-s} \in R^{N_{s} \times N}$ ) whose rows generate the subspace spanned by the eigenvectors corresponding to the positive eigenvalues of $G_{A}$ at the steady solution $A_{+}$(respectively, to the negative eigenvalues of $G_{A}$ at $A_{-}$). Here $N_{u}$ (respectively, $N_{s}$ ) is the number of positive eigenvalues of $G_{A}$ at the point $A_{+}$(respectively, the negative ones at $A_{-}$). Then the truncated problem defined on the interval $\mathcal{T}$ is

$$
\begin{gathered}
\frac{d A(t)}{d t}=G(A(t), \lambda), \quad t \in \mathcal{T}, \\
L_{+u}\left(A\left(T_{+}\right)-A_{+}\right)=0, \\
L_{-s}\left(A\left(T_{-}\right)-A_{-}\right)=0, \\
\Psi(A)=0 .
\end{gathered}
$$

The boundary conditions (4.4), (4.5) in the above system are called projected boundary conditions. Condition (4.4) is a projection onto the stable manifold when $t \rightarrow+\infty$, while (4.5) is a projection onto the unstable manifold of $A_{+}$when $t \rightarrow-\infty$. Such a system was introduced by Beyn in [1] and [2] for the purpose of computing connections for ODEs following their introduction in a broader context by [22] and [24] and, more recently by [15] and [16]. Similar methods were discussed for connecting orbit problems by Doedel and colleagues in, for example, [9], [14], [11], and [10]. $\Psi(A)$ denotes the phase-fixing condition, together with any determining conditions (if necessary). The determining conditions are not required in [1] and [2], since those papers are concerned with the computation of isolated connections. We detail our choices for these extra conditions below.

A suitable choice for the truncation interval $\mathcal{T}=\left[T_{-}, T_{+}\right]$is not easy to determine, in general, except as part of some continuation process. However, in some special cases, we can obtain reasonable estimates for it, as we now describe. For any fixed parameter $\lambda=\lambda_{0}, A_{-}$and $A_{+}$are on particular steady-state branches of (4.1). From the results of Proposition 3.4, we know that the eigenvalues of $G_{A}\left(A_{+}, \lambda_{0}\right)$ are real and that there is a negative eigenvalue $\mu^{+}$of smallest magnitude. Similarly, there is a positive eigenvalue of $G_{A}\left(A_{-}, \lambda_{0}\right)$ of smallest magnitude, denoted $\mu^{-}$. Suppose that $A(t)$ is a solution of the system of (4.3), and that it approaches $A_{+}$when $t \rightarrow+\infty$, where $A_{+}$is a steady solution of (4.3). Linearizing about $A_{+}$, we obtain

$$
\frac{d\left(A(t)-A_{+}\right)}{d t} \approx G_{A}\left(A_{+}, \lambda_{0}\right)\left(A(t)-A_{+}\right) .
$$

If the tolerance we are using in the numerical computation is $\epsilon$, then, arguing from the slowest decay rates, we require that $e^{\mu^{+} T_{+}}<\epsilon$, and thus we obtain the lower bound

$$
T_{+}>-\log (\epsilon) / \mu^{+} \text {. }
$$

Similarly, we can obtain the following result for $T_{-}$:

$$
T_{-}<\log (\epsilon) / \mu^{-} \text {. }
$$


The estimates given by (4.8) and (4.9) give a lower bound for an appropriate truncated interval. When considering connections that approach other equilibria between the $\alpha$ and $\omega$ limit sets or that exhibit metastability [5], these estimates are not suitable, and longer time intervals are required.

Before describing specific computational cases, we now discuss the determining conditions. For (4.3)-(4.6), to define a well-posed BVP, we require $N$ boundary conditions. For sufficiently accurate spectral approximation (sufficiently large $N$ ), $N_{u}=\operatorname{index}(\psi)$, since the largest eigenvalues of the Frechet derivative of $(2.11)$ converge under spectral approximation [7]. Similarly, for large $N, N_{s}=N-\operatorname{index}(\phi)$, Thus, together with the phase condition from (4.6), conditions (4.4), and (4.5) yield

$$
N+1+\operatorname{index}(\phi)-\operatorname{index}(\psi)
$$

boundary conditions. From Theorem 2.10, however, the dimension of the manifold of heteroclinic connections is given by $(2.13)$. Hence $(4.10)$ gives

$$
N+1-\operatorname{dim}\left[W^{u}(\phi) \cap W^{s}(\psi)\right]
$$

boundary conditions. Thus, if the connections have dimension 1 (i.e., a unique connection, up to phase shifts), no extra boundary conditions are required. However, if the connections have dimension $m$ (i.e., an $m-1$ parameter family of connections in $H_{0}^{1}$, up to phase shifts), then $m-1$ extra boundary conditions are required.

For the Chafee-Infante problem, the value of $m$ can be determined by formulae (2.14), (2.15). We now discuss suitable choices for the extra $m-1$ boundary conditions, noting that these conditions represent parameterization of the global attractor. We concentrate on problem (2.1)-(2.4), together with the supplementary condition (F4).

4.1. Connections with $\omega$-limit set on the first branch. Since the first branch $u_{1}^{ \pm}(\lambda)$ (see Fig. 2.1, §2) is stable both theoretically (Theorem 2.9) and numerically, we have $N_{u}=0$. Hence the boundary condition (4.4) is redundant. Such connections are simple computationally, since it is only necessary to solve the initial value problem (4.3) together with initial data $A\left(t_{0}\right)$; see Remark 3.5(ii). For almost all initial data $A(t, 0)$, the solution $A(t)$ approaches $A_{+}$, an approximation to $u_{1}^{+}(\lambda)$ or $u_{1}^{-}(\lambda)$ as $t \rightarrow \infty$. (For the PDE, the initial data on the stable manifolds of $u_{1}^{ \pm}$is dense in $H_{0}^{1}[21]$.) Hence, for any fixed parameter $\lambda=\lambda_{0}$, we consider the following initial value problems:

$$
\begin{gathered}
\frac{d A(t)}{d t}=G\left(A(t), \lambda_{0}\right), \\
A\left(t_{0}\right)=A_{-}+\sum_{j=1}^{N-N_{s}} \epsilon_{j} \gamma_{j} .
\end{gathered}
$$

Here, $\gamma_{j}, j=1,2, \ldots, N-N_{s}$ are orthonormalized eigenvectors of $G_{A}\left(A_{-}, \lambda_{0}\right)$ that correspond to its positive eigenvalues, and $\epsilon_{j}$ are real, positive, and small numbers. We normalize the $\epsilon_{j}$ so that

$$
\sum_{j=1}^{N-N_{s}} \epsilon_{j}^{2}=\epsilon \ll 1
$$

Hence we have $\left(N-N_{s}-1\right)$ free parameters to vary, which represent parameterization of the attractor. These are the determining conditions in this case. 
4.2. Connections inside the invariant subspaces. Using Theorem 2.7 , we restrict initial data to the invariant subspace $\mathbf{Z}_{k}$. In this subspace, the first branch of bifurcating solutions is $u_{k}^{ \pm}(\lambda)$, and, by Proposition 2.8 , this solution is stable in $\mathbf{Z}_{k}$. If we apply the restricted Galerkin expansion given by (3.18), then the same arguments as in $\S 4.1$ may be used to compute those connections with $\omega$-limit set on $u_{k}^{ \pm}(\lambda) \in \mathbf{Z}_{k}$, purely as solutions of initial value problems.

These computations provide starting points for the continuation methods we now describe to approximate connections that are not attainable as solutions of initial value problems.

4.3. General connections. For most connections, it is not possible simply to solve an initial value problem, and the BVP (4.3)-(4.6) must be tackled directly. For this, we use the package AUTO [9], [11], and so we rescale the problem onto a unit time interval as follows:

$$
\begin{gathered}
\frac{d A(t)}{d t}=\rho G(A(t), \lambda), \quad t \in[0,1] \\
L_{+u}\left(A(1)-A_{+}\right)=0 \\
L_{-s}\left(A(0)-A_{-}\right)=0 \\
\Psi_{p}(A)=0 \\
\Psi_{d}(A)=0
\end{gathered}
$$

where $\rho$ denotes the time-rescaling parameter and is usually chosen adaptively during any continuation procedure. Here $\Psi_{p}(A)$ is the phase condition, and $\Psi_{d}(A)$ defines the determining condition(s). It is known that the package AUTO is less sensitive to integral (nonlocal) conditions than to local ones, and so, for the phase condition, we take

$$
\Psi_{p}(A)=\int_{0}^{1}(A(t)-\tilde{A}(t))^{T} G(A(t), \lambda) d t
$$

where $\tilde{A}(t)$ is the solution at the last continuation step solution in that system; such a choice is recommended in [10].

If the connections form a manifold of dimension $m>1$, we require $(m-1)$ extra determining conditions (as argued above). We then use these extra conditions to perform continuation on the attractor. A starting point for the numerical continuation may be obtained from the initial value methods of $\S \S 4.1$ and 4.2 , by working in an appropriate subspace. We describe the particular choices for $\Psi_{d}(A)$ in the $\S 5$ where specific connections are computed. As for the phase condition, we implement $\Psi_{d}(A)$ as an integral condition over time.

Finally, we note that in all the numerical results described in $\S \S 5$ and 6 , we took $N=20$; that is, we used twenty Fourier basis functions in the Galerkin method. This choice is adequate, and the results are robust to increases in $N$.

5. Numerical results for the Chafee-Infante problem. Here we concentrate on (2.1)-(2.4) with the choice $f(u)=u^{3}-u$. Figure 2.1 shows the steady-state solutions computed using the restricted Galerkin spectral expansion (3.18) based on the invariant subspaces $\mathbf{Z}_{k}$ defined by (2.12). Throughout the following, we use the notation $u_{k}^{ \pm}(\lambda)$ as detailed in Theorem 2.9 for steady solutions of the problem. In the heading of the subsections, $\alpha$ and $\omega$ denote the $\alpha$ and $\omega$ limit sets of the computed heteroclinic orbit, respectively. 
(a)

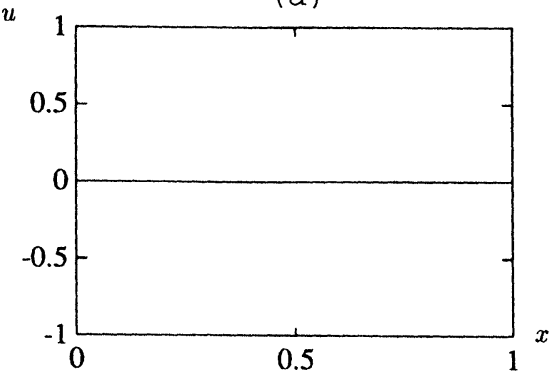

$u$

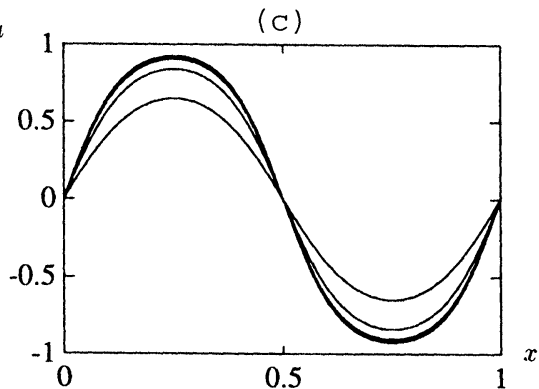

(b)
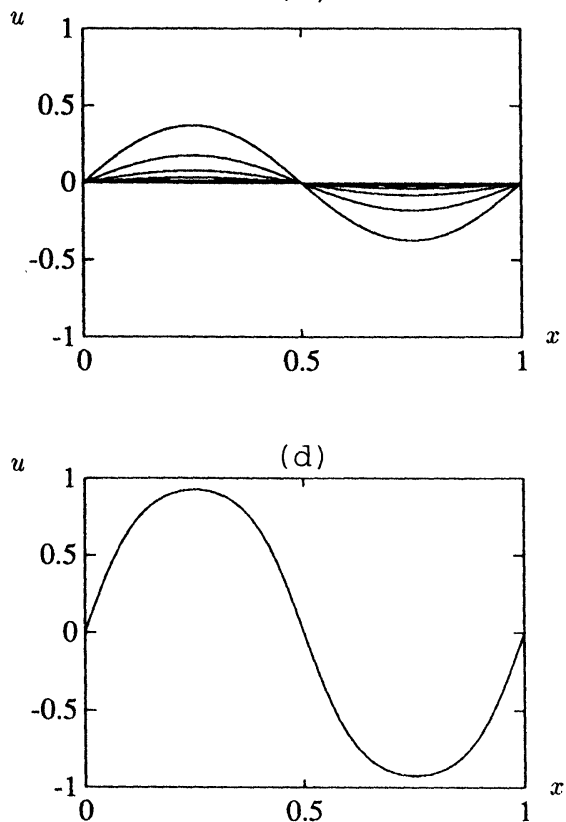

FIG. 5.1. $\lambda=130, \mu=0$. The time interval is (a) $0.0 \leq t \leq 0.3967$, (b) $0.3967 \leq t \leq 0.4667$, (c) $0.4667 \leq t \leq 0.5967$, and (d) $0.5967 \leq t \leq 0.9$.

5.1. $\alpha(u(0))=0, \omega(u(0))=u_{2}^{ \pm}(\lambda), \lambda=130$. For $\lambda=130$, we have $9 \pi^{2}<\lambda<$ $16 \pi^{2}$. Thus, by Theorem 2.10 , the connections between 0 and $u_{2}^{ \pm}(\lambda)$ form a manifold of dimension 2 . Then, by (4.11), we require one extra determining condition. For this, we take

$$
\Psi_{d}(A):=\int_{0}^{1}\left[a_{3}(t)\right]^{2} d t-\mu .
$$

Initially setting $\mu=0$, we obtain solutions for which $a_{3}(t) \equiv 0$ for all $t$. These represent solutions of the PDE that lie in the invariant subspace $\mathbf{Z}_{2}$ defined by (2.12) and that may be found by the initial value techniques in $\S 4.2$. Such a solution is shown in Figs. 5.1 and 5.2. Figure 5.1 shows solution profiles $u(x, t)$ against $x$ at various times, and the connection from the trivial solution to $u_{2}^{+}(x)$ is clear. Note the antisymmetry of the solution profiles about $x=\frac{1}{2}$, which follows from working in $\mathbf{Z}_{2}$. This is reflected in Fig. 5.2(c), where the midpoint $u\left(\frac{1}{2}, t\right)$ is plotted as a function of $t$ and clearly seen to be zero. Now we take $\mu$ as a continuation parameter, and, for nonzero $\mu$, the connections do not lie in an invariant subspace so it is necessary to solve the BVP to find connections. For large values of $\mu$, the connections between the trivial solution and $u_{2}^{+}(x)$ approach the third solution branch before finally reaching the second branch at $t=+\infty$. Such a solution is illustrated in Figs. 5.3 and 5.4, for $\mu=0.8622 \times 10^{-1}$. In particular, Fig. $5.3(\mathrm{~b})$ clearly shows that the solution is very close to the third branch. Figure $5.4(\mathrm{c})$ shows how the antisymmetry for $\mu=0$ is broken for $\mu \neq 0$.

As $\mu$ increases, we find that the connection from zero to $u_{2}^{+}$becomes closer and closer to the union of a connection from zero to $u_{3}^{+}$and a connection from $u_{3}^{+}$to $u_{2}^{+}$. It is possible to approximate these two connections directly, and this has been done. 
$\|u\|_{\infty}$

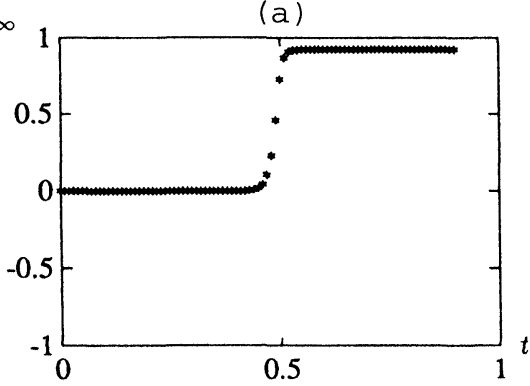

$u\left(\frac{1}{2}\right)$

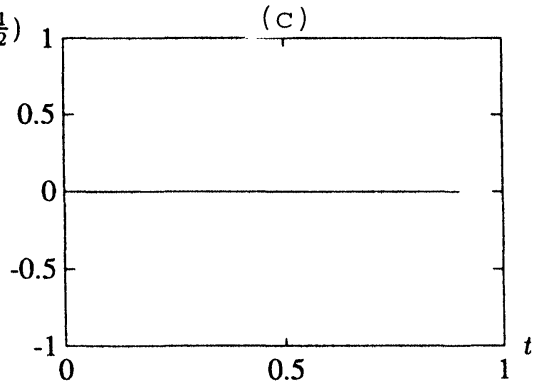

$u\left(\frac{1}{4}\right)$

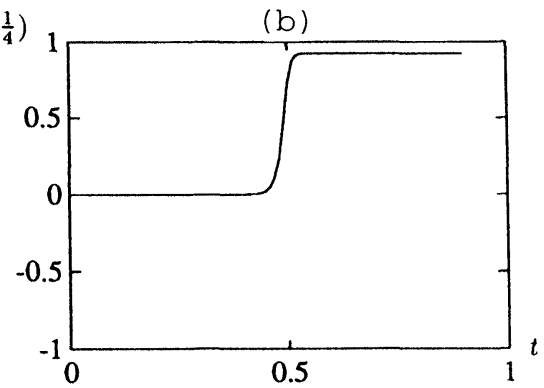

(d)

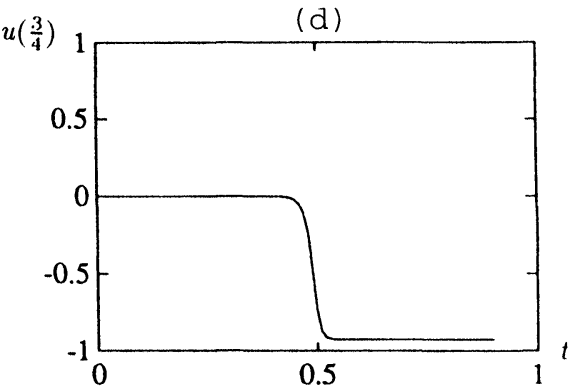

FIG. 5.2. $\lambda=130, \mu=0$. The argument of $u$ denotes the $x$ variable. (a) indicates the distribution of mesh points in time, which is uniform here.

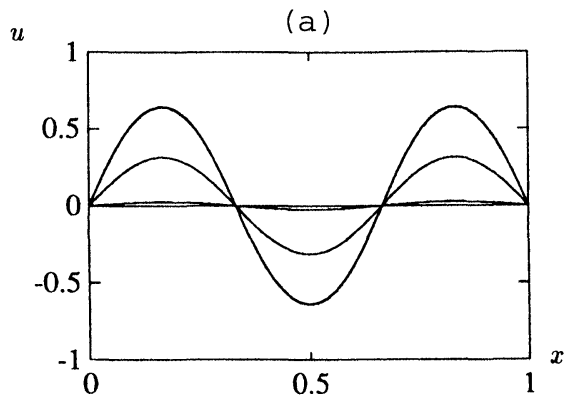

(c)

$u$

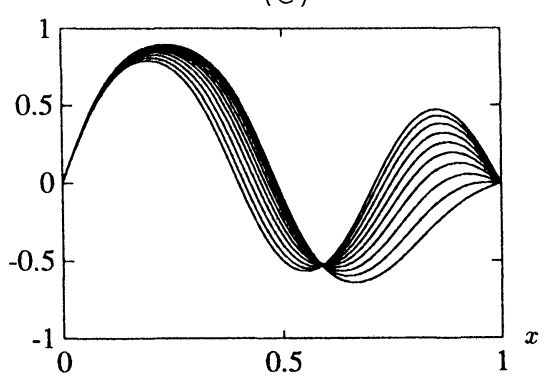

(b)

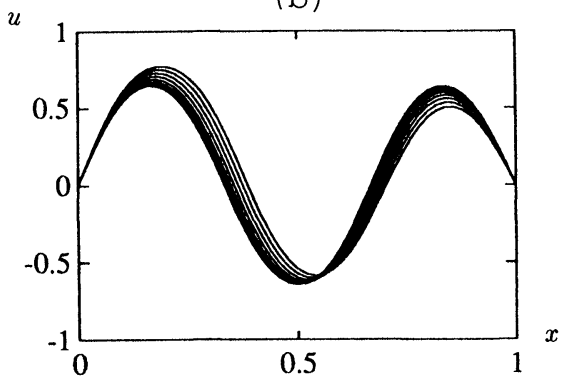

(d)

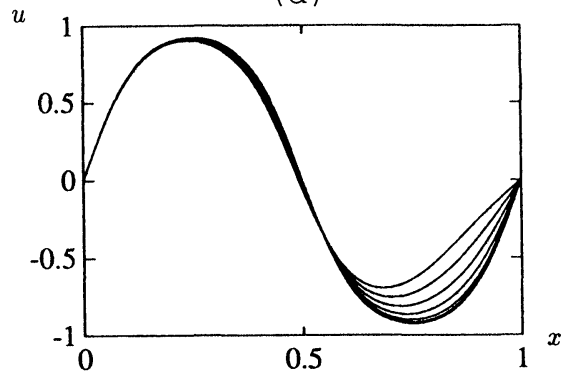

FIG. 5.3. $\lambda=130, \mu=8.622 \times 10^{-2}$. The time interval is (a) $0.0 \leq t \leq 1.4719$, (b) $1.4719 \leq t \leq 1.6837$, (c) $1.6837 \leq t \leq 1.7324$, and (d) $1.7324 \leq t \leq 3.2279$. 

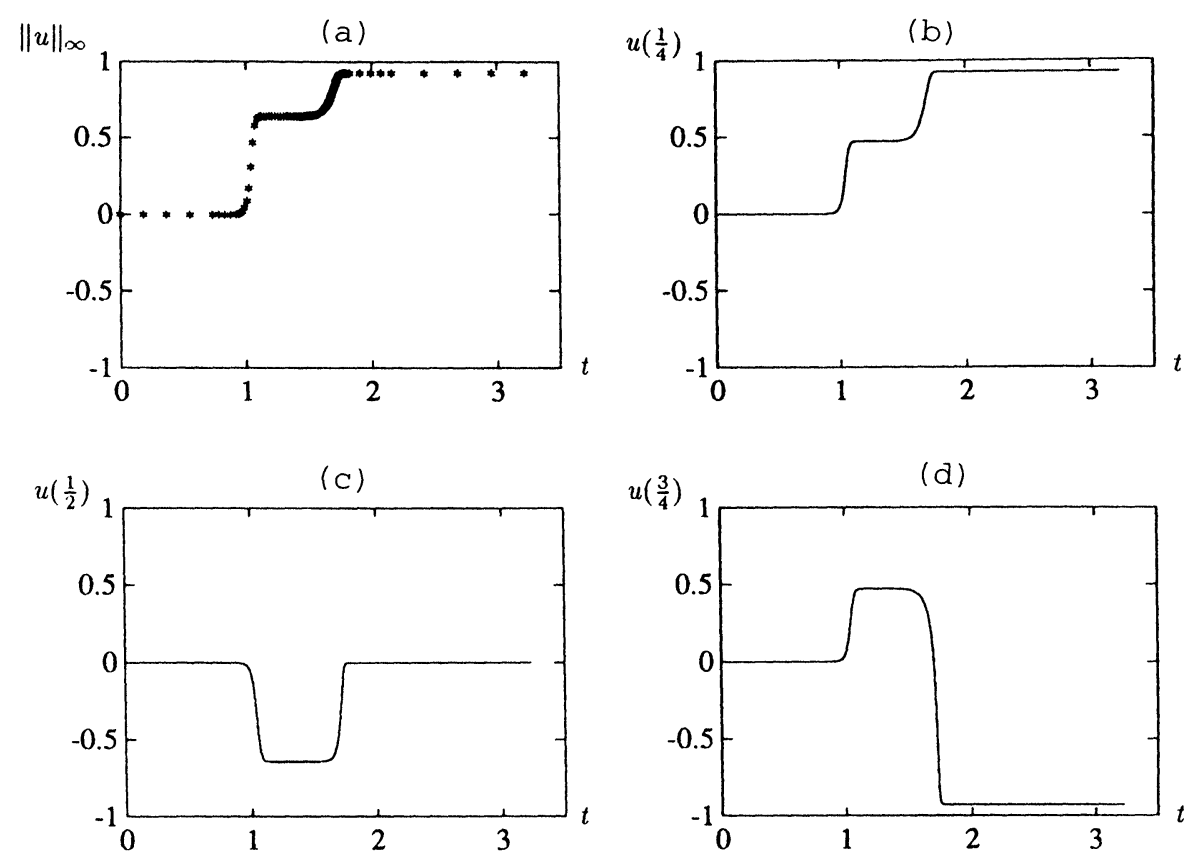

FIG. 5.4. $\lambda=130, \mu=8.622 \times 10^{-2}$. The argument of $u$ denotes the $x$ variable. (a) indicates the distribution of mesh points in time at this continuation step.

Throughout the continuation, the time rescaling parameter $\rho$ is chosen as a function of $\mu$ such that $\rho(\mu)$ is monotonically increasing with $\mu$.

5.2. $\alpha(u(0))=0, \omega(u(0))=u_{2}^{ \pm}(\lambda), \lambda=190$. For $\lambda=190$, we have $16 \pi^{2}<\lambda<$ $25 \pi^{2}$. Thus, by Theorem 2.10 , the connections between 0 and $u_{2}^{ \pm}(\lambda)$ form a manifold of dimension 3. Then, by (4.11), we require two extra determining conditions. For these, we take

$$
\begin{aligned}
& \Psi_{d}^{1}(A)=\int_{0}^{1}\left[a_{3}(t)\right]^{2} d t-\mu_{1}, \\
& \Psi_{d}^{2}(A)=\int_{0}^{1}\left[a_{4}(t)\right]^{2} d t-\mu_{2} .
\end{aligned}
$$

As before, we compute an initial approximation by working in an invariant subspace in which $u_{2}^{ \pm}$are stable and then solve an initial value problem. The subspace we take here is

$$
\operatorname{span}\{\sin (2 j \pi x) \mid j \in \mathbf{N}\} .
$$

In this subspace, the trivial solution zero at $\lambda=190$ has a two-dimensional unstable manifold spanned by $\{\sin (2 \pi x), \sin (4 \pi x)$,$\} ; by taking initial data that is significantly$ larger in $\sin (4 \pi x)$, than in $\sin (2 \pi x)$, we find an initial approximation that passes close to one of the fourth branches $u_{4}^{ \pm}$before ending up at $u_{2}^{+}$. Such a connection corresponds to $\mu_{1}=0$ and nonzero $\mu_{2}$; see Figs. 5.5 and 5.6. Having found this initial approximation, we perform continuation in the parameter $\mu_{1}$ to find solutions which pass close to both the fourth and third branches of steady solutions before reaching the $\omega$ limit set $u_{2}^{+}$. Such a connection is shown in Figs. 5.7 and 5.8. 

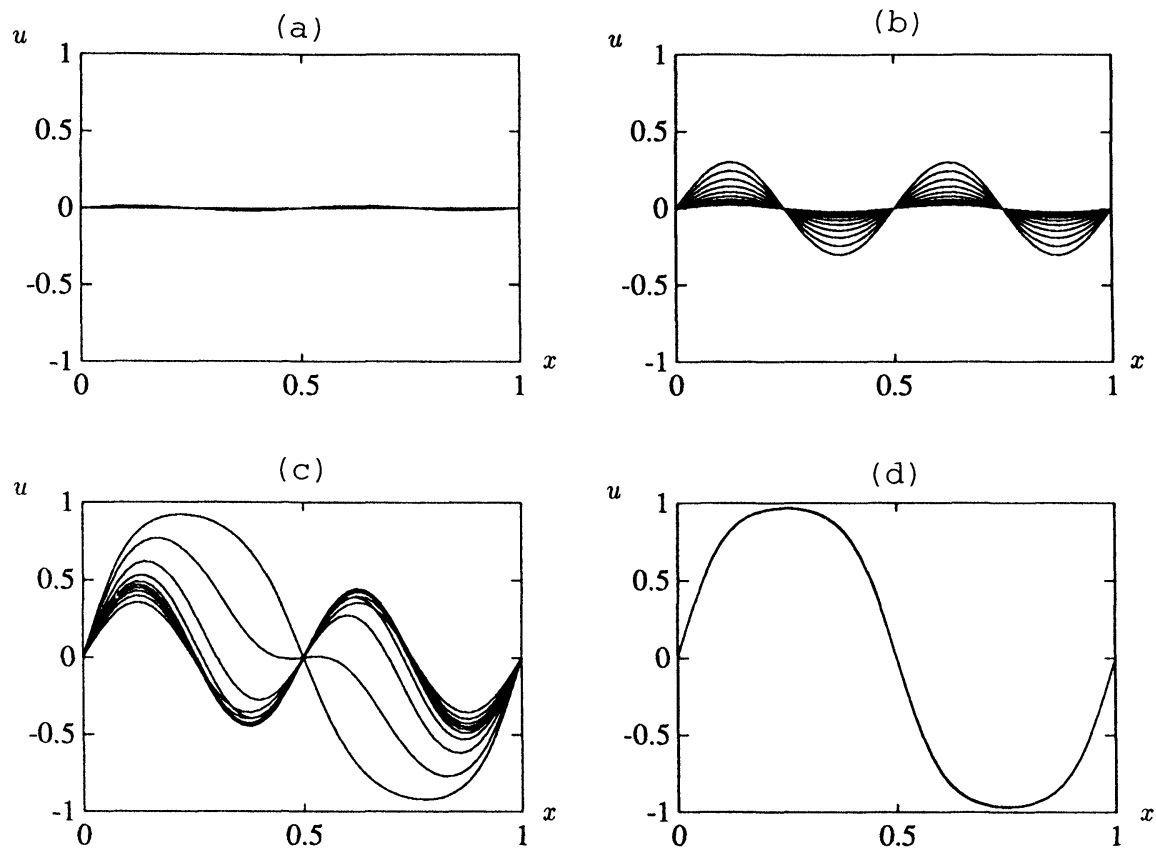

FIG. 5.5. $\lambda=190, \mu_{1}=0, \mu_{2}=1.85034 \times 10^{-2}$. The time interval is (a) $0.0 \leq t \leq 0.3967$, (b) $0.3967 \leq t \leq 0.4667$, (c) $0.4667 \leq t \leq 0.5967$, and (d) $0.5967 \leq t \leq 1.0$.
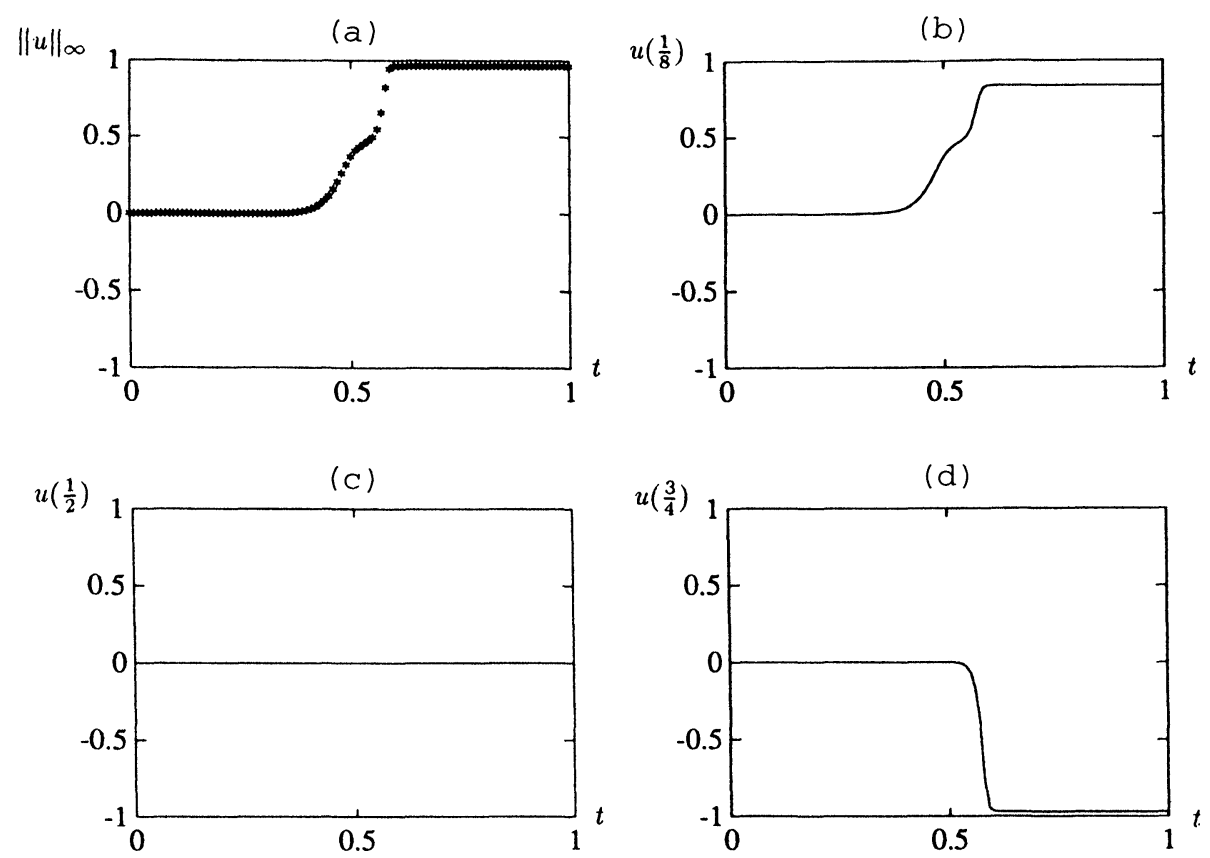

FIG. 5.6. $\lambda=190, \mu_{1}=0, \mu_{2}=1.85034 \times 10^{-2}$. The argument of $u$ denotes the $x$ variable. (a) indicates the distribution of mesh points in time, which is uniform here. 
(a)

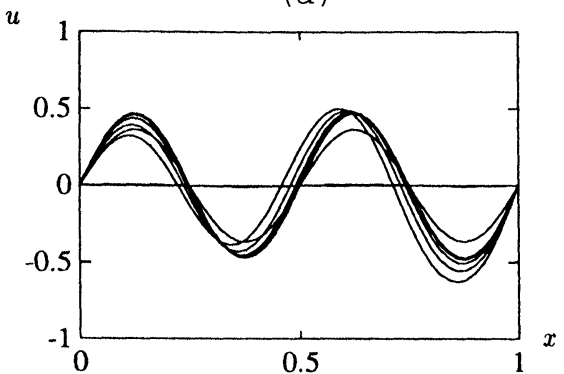

(c)

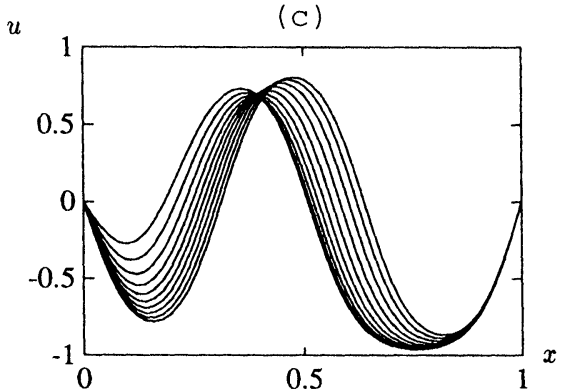

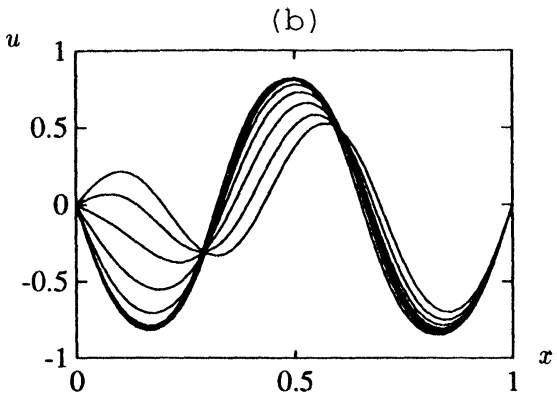

(d)

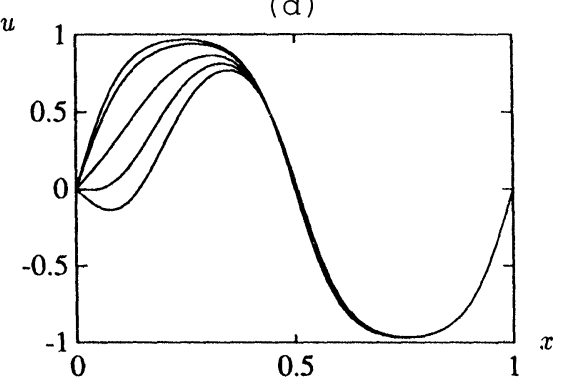

FIG. 5.7. $\lambda=190, \mu_{1}=9.672 \times 10^{-2}, \mu_{2}=1.85034 \times 10^{-2}$. The time interval is (a) $0.0 \leq t \leq 1.7167$, (b) $1.7167 \leq t \leq 2.0398$, (c) $2.0398 \leq t \leq 2.2477$, and (d) $2.2477 \leq t \leq 3.5121$.
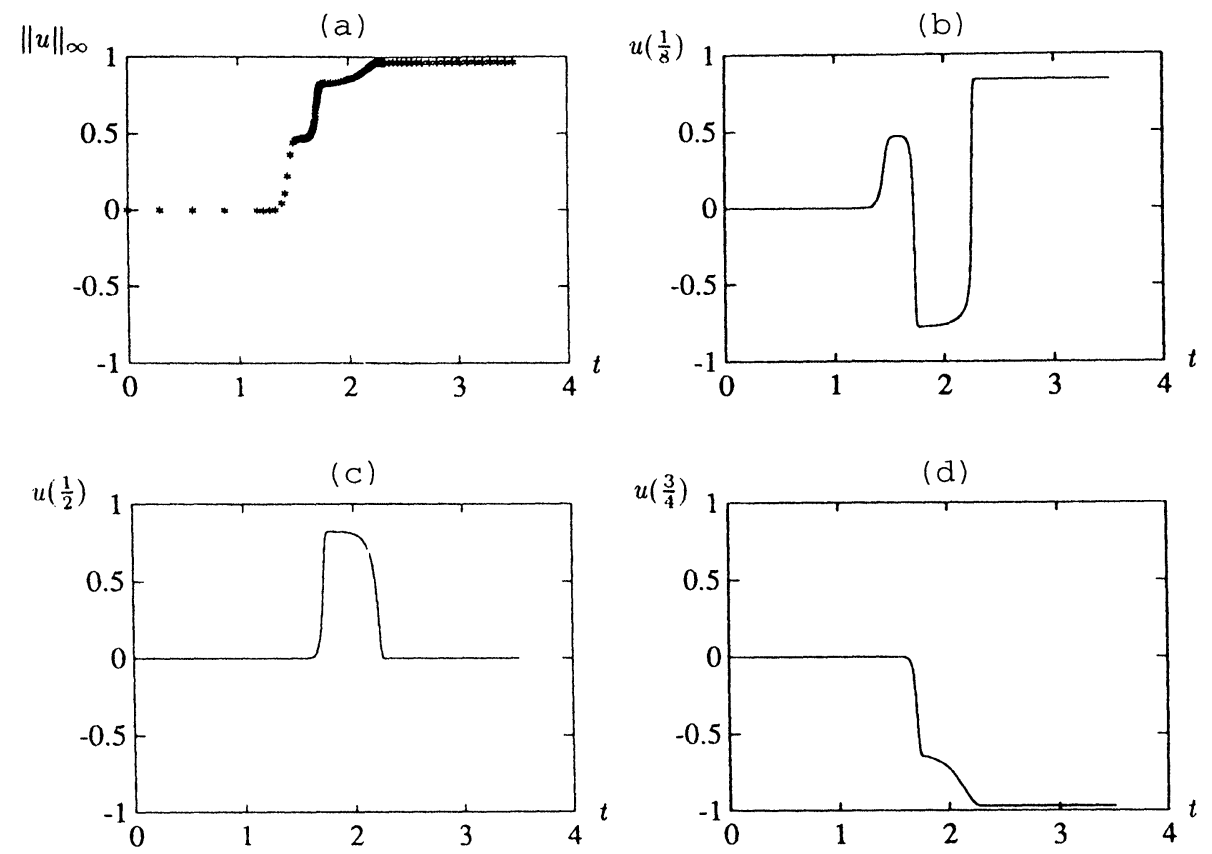

FIG. 5.8. $\lambda=190, \mu_{1}=9.672 \times 10^{-2}, \mu_{2}=1.85034 \times 10^{-2}$. The argument of $u$ denotes the $x$ variable. (a) indicates the distribution of mesh points in time at this continuation step. 


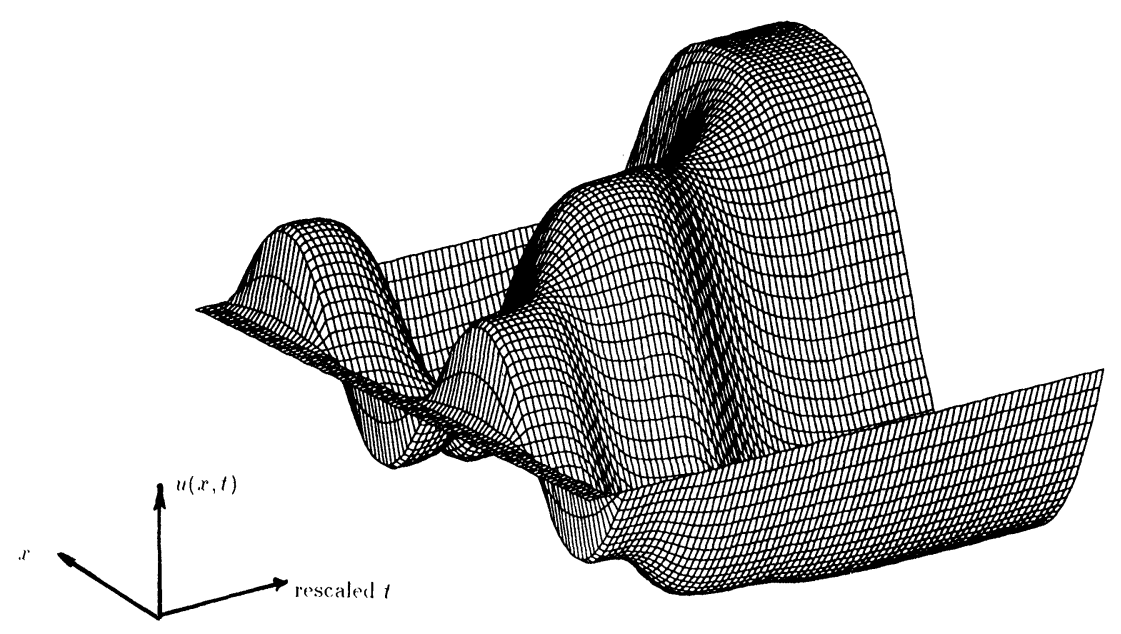

FIG. 5.9. $\lambda=190, \mu_{1}=9.672 \times 10^{-2}, \mu_{2}=1.85034 \times 10^{-2}$. Three-dimensional view of the heteroclinic orbit.

Finally, we show a three-dimensional graph of a heteroclinic orbit to emphasise the fully time-dependent nature of the connections we are computing. Figure 5.9 involves the same data as Figs. 5.7 and 5.8, but is three-dimensional. Note that "time" in Fig. 5.9 has been rescaled according to the distribution of meshpoints indicated in Fig. 8(a).

6. Numerical results for the Cahn-Hilliard equation. In this section, we describe numerical results for the Cahn-Hilliard equation

$$
u_{t}=-\left(u_{x x}-\lambda f(u)\right)_{x x}, \quad x \in(0,1), \quad t>0,
$$

together with the boundary conditions

$$
u(0, t)=w(1, t)=u(1, t)=w(1, t)=0, \quad t>0,
$$

where $w:=-u_{x x}+\lambda f(u)$, and appropriate initial conditions. We assume that $f(u)$ is given by (2.4).

For Neumann boundary conditions, existence and uniqueness is proved in [12], and the existence of absorbing sets and attractors is described in [26]. The equation we consider does not have the physical significance of the equation with Neumann boundary conditions. However, we study here the interesting mathematical question of whether the global attractor for the Chafee-Infante problem (2.1)-(2.4) can be continuously deformed into that for the Cahn-Hilliard equations (6.1), (6.2) - note that both these problems share the same set of equilibria. To this end, we introduce the family of PDEs

$$
u_{t}=-\gamma\left(u_{x x}-\lambda f(u)\right)_{x x}+(1-\gamma)\left(u_{x x}-\lambda f(u)\right), \quad x \in(0,1), \quad t>0
$$

together with the boundary conditions

$$
u(0, t)=w(1, t)=u(1, t)=w(1, t)=0, \quad t>0
$$


and appropriate initial conditions. Note that, for $\gamma=0$, we obtain the ChafeeInfante problem, while, for $\gamma=1$, we obtain the Cahn-Hilliard equation; for any $0 \leq \gamma \leq 1$, the equation has a gradient structure. It is now possible, given an approximation to the Chafee-Infante problem, to perform numerical continuation in $\gamma$ to determine whether there is a simple homotopy connecting the global attractors of the two problems.

Practical aspects of the continuation in $\gamma$ are relatively straightforward, and we only make two observations about this.

Remark 6.1. (i) To compute connections from the trivial solution to a nontrivial $\omega$ limit set, we require the eigenvectors spanning the unstable manifold of the $\omega$ limit set, and these depend on $\gamma$. However, numerical computation reveals that the eigenvectors spanning the unstable manifold are insensitive to $\gamma$, and so we simplify the computations by approximating the spanning eigenvectors simply by linear interpolation between the Chafee-Infante and Cahn-Hilliard equations.

(ii) When using AUTO, the BVP defining connections is rescaled onto the unit interval as in (4.12)-(4.16). This introduces the time rescaling parameter $\rho$. A simple argument based on a one-Fourier component Galerkin method indicates that the behaviour of $\rho^{-1}$ is linear in $\gamma$, and so we take

$$
\rho(\gamma)=\frac{\rho(C I) \rho(C H)}{(1-\gamma) \rho(C H)+\gamma \rho(C I)}
$$

where $\rho(C I)$ and $\rho(C H)$ denote appropriate values of $\rho$ for the Chafee-Infante and Cahn-Hilliard problems, respectively. This choice is successful in all our computations.

Numerically, we find that continuation in $\gamma$ leads in a straightforward way from the global attractor of the Chafee-Infante problem to that of the Cahn-Hilliard equation and that, geometrically, there is little to distinguish the two attractors. We describe some specific results for the case where $\lambda=190$.

6.1. $\alpha(u(0))=0, \omega(u(0))=u_{2}^{ \pm}(\lambda), \lambda=190$. For this value of $\lambda$, we compute a solution of the Chafee-Infante problem by the methods described in $\S 5.2$, taking $\mu_{1}=5.4979 \times 10^{-2}$ and $\mu_{1}=1.8503 \times 10^{-2}$. We then perform continuation in $\gamma$, as described above. As in $\S 5.2$, the orbit starts at the trivial solution and then approaches the fourth and third steady-state branches before reaching the $\omega$ limit set on the second branch. Figures 6.1-6.4 show numerical results for the connecting orbit in the two cases where $\gamma=0.5$ and $\gamma=1$. Note that the connections are barely distinguishable in their geometry but that the timescales of the two problems differ by a factor of about two.

Similar calculations have been performed for the Cahn-Hilliard equations for other parameter values $\lambda$; the results are similar and show a continuous deformation from the Chafee-Infante problem. The study of (6.3) together with homogeneous Neumann boundary conditions is the subject of current investigations. 
$u$

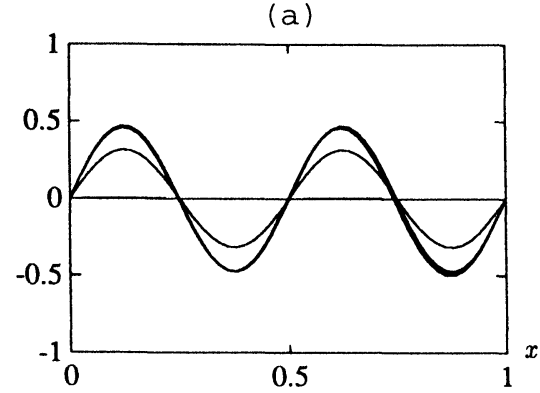

(c)

$u$

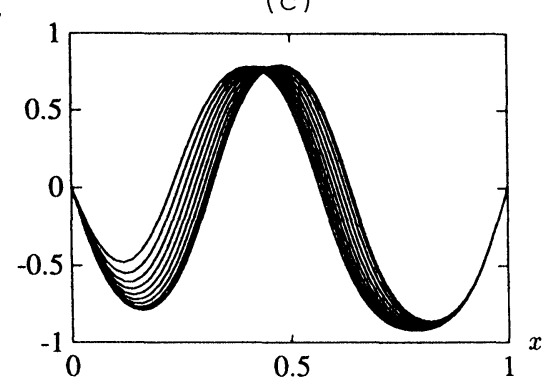

(b)

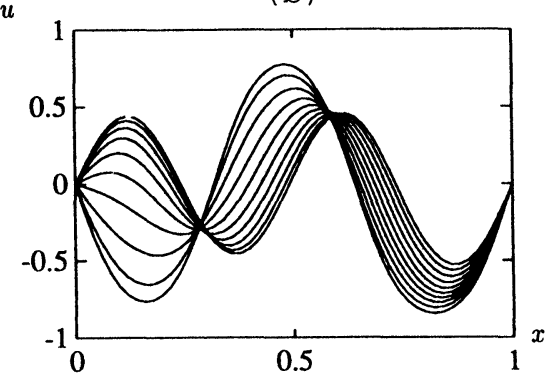

(d)

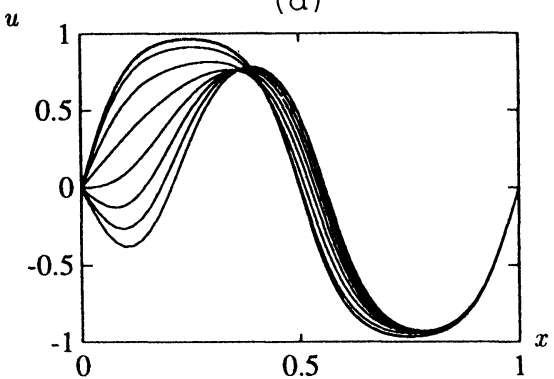

FIG. 6.1. $\lambda=190, \mu_{1}=5.4979 \times 10^{-2}, \mu_{2}=1.8503 \times 10^{-2}, 4 \gamma=0.5$. The time interval is (a) $0.0 \leq t \leq 0.0447$, (b) $0.0447 \leq t \leq 0.0466$, (c) $0.0466 \leq t \leq 0.0536$, and (d) $0.0536 \leq t \leq 0.0875$.

$\|u\|_{\infty}$
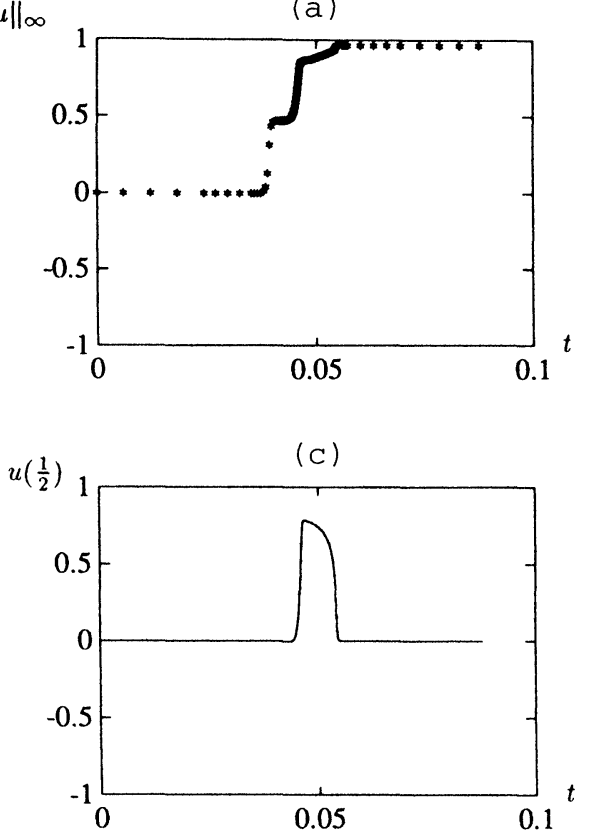

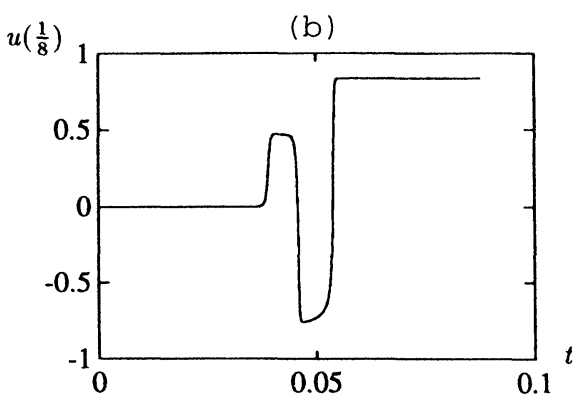

(d)

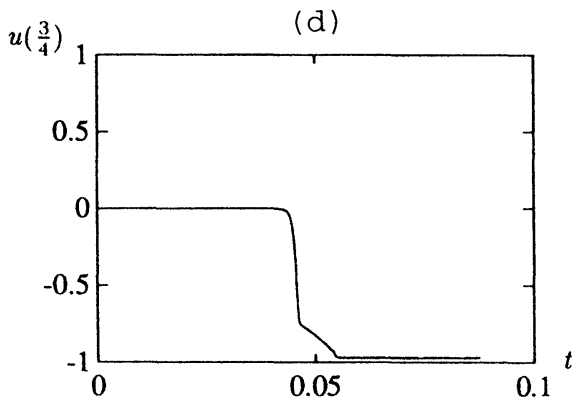

FIG. 6.2. $\lambda=190, \mu_{1}=5.4979 \times 10^{-2}, \mu_{2}=1.8503 \times 10^{-2}, \gamma=0.5$. The argument of $u$ denotes the $x$ variable. (a) indicates the distribution of mesh points in time. 
$u$

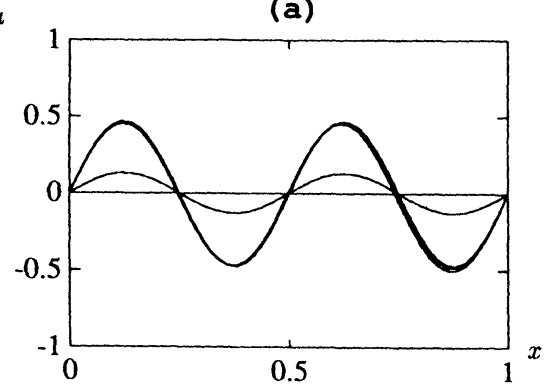

(c)

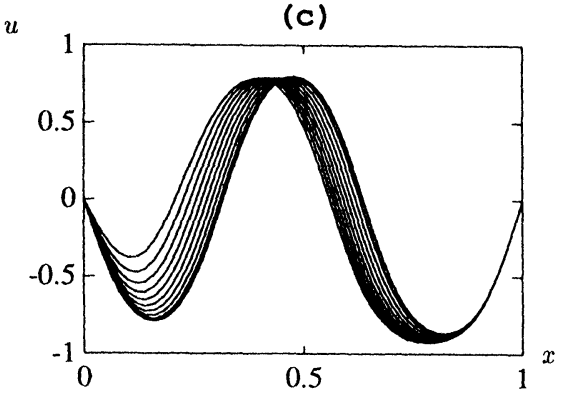

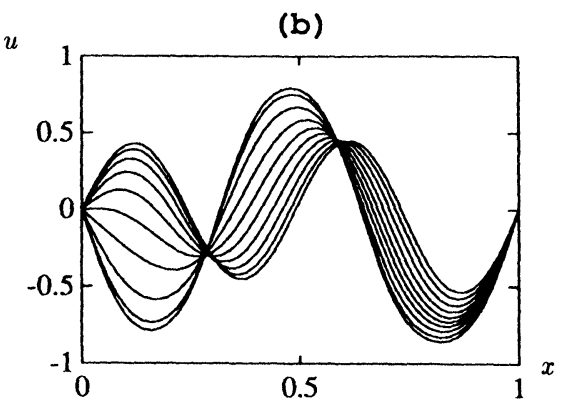

(d)

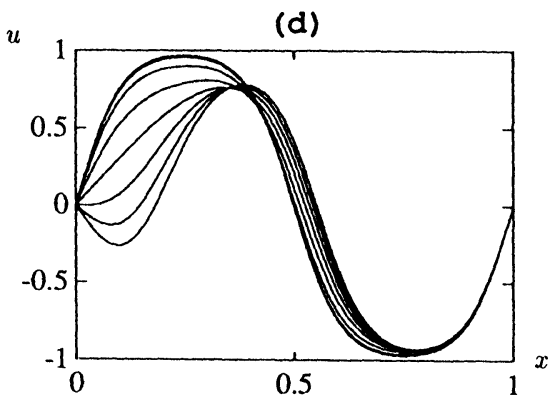

FIG. 6.3. $\lambda=190, \mu_{1}=5.4979 \times 10^{-2}, \mu_{2}=1.8503 \times 10^{-2}, \gamma=1.0$. The time interval is (a) $0.0 \leq t \leq 0.0228$, (b) $0.0228 \leq t \leq 0.0240$, (c) $0.0240 \leq t \leq 0.0274$, and (d) $0.0274 \leq t \leq 0.0446$.
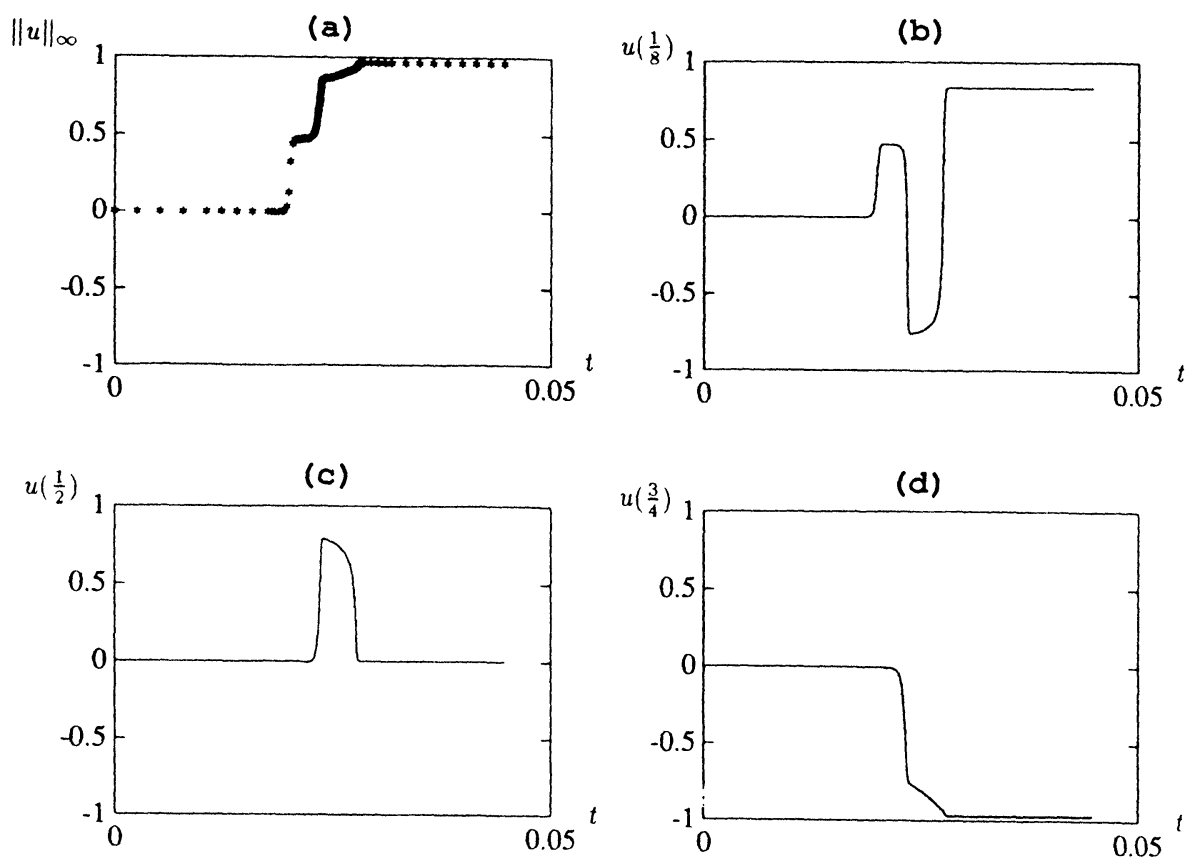

FIG. 6.4. $\lambda=190, \mu_{1}=5.4979 \times 10^{-2}, \mu_{2}=1.8503 \times 10^{-2}, \gamma=1.0$. The argument of $u$ denotes the $x$ variable. (a) indicates the distribution of mesh points in time. 
Acknowledgments. Fengshan Bai is grateful to Professor E. Doedel for helpful comments and suggestions on the use of the package AUTO and to the United Kingdom Science Engineering Research Council with respect to a postdoctoral research position.

\section{REFERENCES}

[1] W. J. BEYN, The numerical computation of connecting orbits in dynamical systems, IMA J. Numer. Anal., 9 (1990), pp. 379-405.

[2] - Global bifurcations and their numerical computation, in Continuation and Bifurcations: Numerical Integration Techniques and Applications, D. Roose and A. Spence, eds., NATO ASI Series, Leuven, Belgium, 1989.

[3] G. Caginalp, An analysis of a phase field model of a free boundary, Arch. Rat. Mech. Anal., 92 (1986), pp. 205-245.

[4] J. W. CAHN AND J. E. HilliaRD, Free energy of a non-uniform system I. Interfacial free energy, J. Chem. Phys., 28 (1958), pp. 258-267.

[5] J. CARR AND R. L. PEgo, Metastable patterns in solution of $u_{t}=\epsilon^{2} u_{x x}-f(u)$, Comm. Pure Appl. Math., 42 (1989), pp. 523-576.

[6] N. Chafee And E. F. Infante, A bifurcation problem for a nonlinear partial differential equation of parabolic type, J. Appl. Anal., 4 (1974), pp. 17-35.

[7] F. Chatelin, Spectral Approximation of Linear Operators, Academic Press, New York, 1983.

[8] S. Chow And J. K. Hale, Methods of Bifurcation Theory, Springer-Verlag, New York, Berlin, Heidelberg, 1982.

[9] E. J. Doedel, Numerical analysis and control of bifurcation problems, Univ. Minnesota Supercomputer Institute Report, 89/17, 1989.

[10] E. J. Doedel And M. J. Friedman, Numerical computation of heteroclinic orbits, J. Comput. Appl. Math., 26 (1989), pp. 159-170.

[11] E. J. Doedel And J. P. Kernẽvez, AUTO: Software for Continuation and Bifurcation Problems in Ordinary Differential Equations, Applied Math. Report, California Institute of Technology, Pasadena, CA, 1986.

[12] C. M. Elliott And Z. Songmu, On the Cahn-Hilliard equation, Arch. Rat. Mech. Anal., 96 (1986), pp. 339-357.

[13] C. M. Elliott And A. M. Stuart, The global dynamics of discrete semilinear parabolic equations, SIAM J. Numer. Anal., to appear.

[14] M. J. Friedman And E. J. Doedel, Numerical computation and continuation of invariant manifolds connecting fixed points, SIAM J. Numer. Anal., 28 (1991), pp. 789-808.

[15] T. HaGstrom AND H. B. Keller, Exact boundary conditions at an artificial boundary for partial differential equations in cylinders, SIAM J. Math. Anal., 17 (1986), pp. 322-341.

[16] - Asymptotic boundary conditions and numerical methods for nonlinear elliptic problems on unbounded domains, Math. Comp., 48 (1987), pp. 449-470.

[17] J. K. Hale, Asymptotic Behavior of Dissipative Systems, Math. Surveys Monographs, 25, American Mathematical Society, Providence, RI, 1988.

[18] J. K. Hale, L. T. Magalhães, and W. M. Oliva, An Introduction to Infinite Dimensional Dynamical Systems-Geometric Theory, Appl. Math. Sci., Vol. 47, Springer-Verlag, New York, 1984.

[19] J. K. HAle AND G. RAUGeL, Lower semicontinuity of attractors of gradient systems and applications, Ann. Mat. Pura. Appl., 4 (1989), pp. 281-326.

[20] D. Henry, Geometric Theory of Semilinear Parabolic Equations, Lecture Notes in Math., Vol. 840, Springer-Verlag, New York, Berlin, 1981.

[21] - Some infinite dimensional Morse-Smale systems defined by parabolic differential equations, J. Differential Equations, 59 (1985), pp. 165-205.

[22] F. R. DE Hoog AND R. WEISS, An approximation theory for boundary value problems on infinite intervals, Computing, 24 (1980), pp. 227-239.

[23] O. Ladyzhenskaya, Attractors for Semigroups and Evolution Equations, Cambridge University Press, London, 1991.

[24] M. LENTINI AND H. B. KELLER, Boundary value problems over semi-infinite interval and their numerical solution, SIAM J. Numer. Anal., 17 (1980), pp. 577-604.

[25] J. SHEN, Long time stability and convergence of fully discrete nonlinear Galerkin methods, Numer. Funct. Anal. Optim., 38 (1990), pp. 201-229.

[26] R. Temam, Infinite-Dimensional Dynamical Systems in Mechanics and Physics, Appl. Math. Sci., Vol. 68, Springer-Verlag, New York, 1988. 